\title{
Algorithmic randomness over general spaces
}

\author{
Kenshi Miyabe*
}

May 16, 2012

\begin{abstract}
The study of Martin-Löf randomness on a computable metric space with a computable measure has had much progress recently. In this paper we study Martin-Löf randomness on a more general space, that is, a computable topological space with a computable measure. On such a space, Martin-Löf randomness may not be a natural notion because there is not a universal test, and Martin-Löf randomness and complexity randomness (defined in this paper) do not coincide in general. We show that $\mathrm{SCT}_{3}$ is a sufficient condition for the existence and the coincidence and study how much we can weaken the condition.
\end{abstract}

Keywords: computable analysis, computable topological space, separation axiom, computable measure, Martin-Löf randomness

\section{Introduction}

What does it mean to say that a binary sequence is "random"? Von Mises [26] tried to give an answer by introducing the concept of a collective with the motivation of formalizing probability. A collective is an actual sequence whose limiting frequency exists and remains the same when one replaces the sequence with a subsequence. Although Wald and Church gave a rigorous mathematical definition of this concept using the notion of computability, Ville [25] showed that there exists a collective that does not satisfy the law of the iterated logarithm, which a random sequence should satisfy. Hence a collective is not a natural randomness notion.

About fifty years later, Martin-Löf [17] introduced another definition of randomness that is called Martin-Löf randomness now. Martin-Löf randomness has many nice properties. In this paper we forcus on the following two important properties.

(i) Martin-Löf randomness has a universal test, that is, only one test is enough to see whether a sequence is random or not.

\footnotetext{
${ }^{*}$ Research Institute for Mathematical Sciences, Kyoto University, kmiyabe@kurims.kyotou.ac.jp
} 
(ii) Martin-Löf randomness has several characterizations by complexity. The initial contributors are Levin [14, 15], Schnorr [21] and Chaitin [4].

It should be noted that, unlike a collective, each Martin-Löf random sequence satisfies the strong law of large numbers (SLLN) and the law of the iterated logarithm (LIL) $[27,16]$. This is important from the view point of probability theory. As a result, Martin-Löf randomness is now regarded as a natural randomness notion.

Martin-Löf randomness can be generalized to a more general space. Computable analysis [30, 3, 33] defined a computable metric space and a computable topological space, which are a metric space and a topological space equipped with computability. Martin-Löf randomness on a computable topological space has studied in the literature such as Zvonkin and Levin [36] and Hertling and Weihrauch [10]. However it has not been well developed. The following should be noted the following comparing to Martin-Löf randomness on Cantor space.

(i) They showed the existence of a universal test. However they use stronger computability of the measure than we use in this paper.

(ii) So far no characterization by complexity has been known.

In contrast the study on Martin-Löf randomness on a computable metric space had much progress recently.

(i) Martin-Löf randomness has a universal test.

(ii) A characterization by complexity of Martin-Löf test was partially given by Gács [8] and was completely showed by Hoyrup and Rojas [11]. although they use a stronger condition than usual.

The notion of probability has a strong relation with randomness while the usual probability theory does not have a rigorous definition of randomness. Since the probability theory is developed on a measure space, we would like to have a natural and mathematical notion of randomness on a computable topological space with a computable measure.

An important question is whether Martin-Löf randomness is a natural randomness notion even on a computable topological space with a computable measure. Martin-Löf randomness seems a natural notion on Cantor space and on a computable metric space with a computable measure while it is doubtful that Martin-Löf randomness on a computable topological space with a computable measure is a natural randomness notion.

The main claim of this paper is that Martin-Löf randomness is a natural randomness notion only when the underlying topological space has a somewhat strong topology. We will show that, on a computable topological space with a computable measure,

(i) Martin-Löf randomness does not have a universal test,

(ii) Martin-Löf randomness is not equivalent to a randomness notion induced by a kind of complexity 
in general.

Another important claim is the following. We define a randomness notion by complexity and call it complexity randomness. The definition of this randomness notion uses "closed" sets (not open sets), which is siginificantly different from the attempts up to now. The author believes that a randomness notion defined by complexity should use closed sets and will explain the reason later.

Another question discussed in this paper is what is the right definition of a computable measure on a computable topological space. on a computable metric space with a computable measure, Hoyrup and Rojas [11] gave the complete characterization by complexity of Martin-Löf randomness. The argument depends on the detailed study of a computable measure on a computable metric space. The definition of complexity randomness also highly depends on the argument on the definition of a computable measure on a computable topological space.

We review some works of the study of computability of measures. On the unit interval, Weihrauch [29] gave natural computability of measures. Schröder [23] generalized it to a computable topological space. Bosserhoff [2] also used the definition.

Another way is to consider the space of measures. The space of measures on a computable metric space is another computable metric space, which is proved by Gács [8]. This observation naturally induces a natural computability of measures, that is, a computable measure is a computable point in the space. In this paper we take this approach and generalize it. We will see that the definition coincides with the definition by Schröder [23].

Edalat [7] used regularity to study computability of measures and, because of it, it is not general enough. Weihrauch et al. [35, 12] defined a computable measurable space but the requirement of computable measures is too strong from our point of view.

The paper is constructed as follows. In Section 2 we review the needed notions from various areas, namely from topology, measure theory, computable analysis, and about computable topological spaces. In Section 3 we study computability of measures on a computable topological space. In Section 4 we show an unnatural property of Martin-Löf randomness on a computable topological space, taht is, Martin-Löf randomness does not have a universal test in general. In Section 5 we define complexity randomness and study when Martin-Löf randomness and complexity randomness coincide.

\section{Preliminaries}

\subsection{General topology}

We review general topology from [34]. A topology on a set $X$ is a class $\tau$ of subsets of $X$, called the open sets, satisfying the following: closed under union, closed under finite intesection and $\emptyset, X \in \tau$. We say $(X, \tau)$ is a topological space. A closed set is the complement of an open set. A base for $\tau$ is a class $\beta \subseteq \tau$ 
such that each element of $\tau$ is the union of elements of $\beta$. A subbase for $\tau$ is a class $\beta^{\prime} \subseteq \tau$ such that the class of all finite intersections of elements of $\beta^{\prime}$ forms a base for $\tau$. A space is second countable if it has a countable base.

The following are some separation axioms. Let $\mathcal{A}$ be the class of closed sets.

$$
\begin{aligned}
& \left.T_{0}:(\forall x, y \in X, x \neq y)(\exists W \in \tau)((x \in W \wedge y \notin W) \vee(x \notin W \wedge y \in W))\right), \\
& T_{1}:(\forall x, y \in X, x \neq y)(\exists W \in \tau)(x \in W \wedge y \notin W), \\
& T_{2}:(\forall x, y \in X, x \neq y)(\exists U, W \in \tau)(U \cap V=\emptyset \wedge x \in U \wedge y \in V), \\
& T_{3}:(\forall x \in X, \forall A \in \mathcal{A}, x \notin A)(\exists U, W \in \tau)(U \cap V=\emptyset \wedge x \in U \wedge A \subseteq V) .
\end{aligned}
$$

We will speak of $T_{i}$-spaces for $i=0,1,2,3$.

For topological spaces $X$ and $Y$, a function $f: X \rightarrow Y$ is continuous iff for each open set $H$ in $Y, f^{-1}(H)$ is open in $X$. The class of all continuous mappings from $X$ to $Y$ is denoted by $C(X, Y)$; if $Y=\mathbb{R}$, then this class is denoted by $C(X)$. The set of all bounded functions in $C(X)$ is denoted by $C_{b}(X)$.

\subsection{Measure theory}

We review measure theory from [1]. An algebra $\mathcal{F}$ of sets is a class of subsets of some fixed set $X$ such that $\emptyset, X \in \mathcal{F}$ and it is closed under union, intersection and complement. An algebra of sets $\mathcal{A}$ is called a $\sigma$-algebra if it is also closed countable union. A real-valued set function $\mu$ on a class of sets $\mathcal{F}$ is called countably additive if $\mu\left(\bigcup_{n=1}^{\infty} A_{n}\right)=\sum_{n=1}^{\infty} \mu\left(A_{n}\right)$ for all pairwise disjoint sets $A_{n}$ in $\mathcal{F}$ such that $\bigcup_{n=1}^{\infty} A_{n} \in \mathcal{F}$. A countably additive set function defined on an algebra is called a measure. A measure is probabilistic if $\mu(X)=1$. In this paper we only consider non-negative probabilistic measures and we use a measure to mean a non-negative probabilistic measure.

On a topological space $X$, the Borel $\sigma$-algebra $\mathcal{B}(X)$ is the $\sigma$-algebra generated by all open sets. The sets in $\mathcal{B}(X)$ are called the Borel sets in the space $X$. The Baire $\sigma$-algebra $\mathcal{B} a(X)$ is the $\sigma$-algebra generated by all sets of the form $\{x \in X: f(x)>0\}$

The sets in $\mathcal{B} a(X)$ is called the Baire sets in the space $X$. The sets of the form $\{x \in X: f(x)>0\}$ where $f \in C(X)$ are called functionally open and their complements are called functionally closed. In a metric space, any closed set is the set of zeros of a continuous function. Hence the Borel and Baire $\sigma$-algebras of a metric space coincide.

A measure on the Borel $\sigma$-algebra $\mathcal{B}(X)$ is called a Borel measure on $X$ and a measure on the Baire $\sigma$-algebra $\mathcal{B} a(X)$ is called a Baire measure. We use $\mathcal{M}(X)$ to mean the set of all (non-negative probabilistic) Borel measures and $\mathcal{M}_{\sigma}(X)$ to mean the set of all (non-negative probabilistic) Baire measures. The weak topology on the space $\mathcal{M}_{\sigma}(X)$ of Baire measures on $X$ is the topology with the base of the sets

$$
W_{G_{1}, \ldots, G_{n}, \epsilon}(\mu)=\left\{\nu \in \mathcal{M}_{\sigma}(X): \nu\left(G_{i}\right)>\mu\left(G_{i}\right)-\epsilon, i=1, \ldots, n\right\},
$$


where $G_{i}=X \backslash f_{i}^{-1}(0), f_{i} \in C(X), \epsilon>0$. The A-topology on the space $\mathcal{M}(X)$ is defined by means of neighborhoods of the form

$$
U(\mu, G, \epsilon)=\{\nu: \mu(G)<\nu(G)+\epsilon\},
$$

where $\mu \in \mathcal{M}(X), G \in \mathcal{O}(X), \epsilon>0$. In a metric space, the A-topology coincides with the weak topology.

\subsection{Computable analysis}

We will use essentially the terminology from [33]. We assume that the readers are familiar with computability on $\Sigma^{*}$, which has been well studied $[24,19,20,5]$.

Let $\Sigma$ be a finite alphabet such that $0,1 \in \Sigma$. By $\Sigma^{*}$ we denote the set of finite words over $\Sigma$, and by $\Sigma^{\omega}$ the set of infinite sequences $p: \mathbb{N} \rightarrow \Sigma$ over $\Sigma, p=(p(0) p(1) \ldots)$. We use the "wrapping function" $\iota: \Sigma^{*} \rightarrow \Sigma^{*}$, $\iota\left(a_{1} a_{2} \ldots a_{k}\right):=110 a_{1} 0 a_{2} 0 \ldots a_{k} 011$. For $u \in \Sigma^{*}$ and $w \in \Sigma^{*} \cup \Sigma^{\omega}$ let $u \ll w$ iff $\iota(u)$ is a subword of $w$. Let $\langle i, j\rangle:=(i+j)(i+j+1) / 2+j$ be the bijective Cantor pairing function on $\mathbb{N}$. We consider standard functions for finite or countable tupling on $\Sigma^{*}$ and $\Sigma^{\omega}$ denoted by $\langle\cdot\rangle$ in [30, Definition 2.1.7].

A partial function is denoted by $f: \subseteq A \rightarrow B$ and a total function is denoted by $f: A \rightarrow B$. Let $Y_{0}, \ldots, Y_{n} \in\left\{\Sigma^{*}, \Sigma^{\omega}\right\}$ and $Y=Y_{1} \times \ldots \times Y_{n}$. A function $f: \subseteq Y \rightarrow Y_{0}$ is computable if for some Type-2 machine $M, f$ is the function $f_{M}$ computed by $M$. Informally, a Type- 2 machine is a Turing machine, which reads from input tapes with finite or infinite inscription, operates on work tapes and write one-way to an output tape. For $Y_{0}=\Sigma^{*}, f_{M}(y)=w$, if a Turing machine $M$ on input $y$ halts with $w$ on the output tape. For $Y_{0}=\Sigma^{\omega}, f_{M}(y)=q$, if $M$ on input $y$ computes forever and writes writes $q \in \Sigma^{\omega}$ on the output tape. On $\Sigma^{*}$ we consider the discrete topology and on $\Sigma^{\omega}$ the topology generated by the base $\left\{w \Sigma^{\omega}: w \in \Sigma^{*}\right\}$ of open sets. Every computable function is continuous.

A notation of a set $M$ is a surjective function $\gamma: \subseteq Y \rightarrow M$ where $Y=\Sigma^{*}$ and a representation where $Y=\Sigma^{\omega}$. An examples is the representation $\rho: \subseteq \Sigma^{\omega} \rightarrow \mathbb{R}$ of the real numbers, which we will define later. A partial function $h: \subseteq Y \rightarrow Y_{0}$ realizes a function $f: \subseteq M \rightarrow M_{0}$ if $f(x)=\gamma_{0} \circ h(y)$ whenever $x=\gamma(y)$ and $f(x) \downarrow$. This means that $h(y)$ is a name of $f(x)$ if $y$ is a name of $x \in \operatorname{dom}(f)$. The function $f$ is called $\left(\gamma, \gamma_{0}\right)$-computable if it has a computable realization. A point $x \in M_{1}$ is $\gamma_{1}$-computable iff $x=\gamma_{1}(p)$ for some computable $p \in \operatorname{dom}\left(\gamma_{1}\right)$.

We write $\gamma_{1} \leq \gamma_{0}\left(\gamma_{1}\right.$ is reducible to $\left.\gamma_{0}\right)$ if $M_{1} \subseteq M_{0}$ and the identity id : $M_{1} \rightarrow M_{0}$ is $\left(\gamma_{1}, \gamma_{0}\right)$-computable. This means that some computable function $h$ translates $\gamma_{1}$-names to $\gamma_{0}$-names, that is, $\gamma_{1}(p)=\gamma_{0} \circ h(p)$. Computable equivalence is defined canonically.

$F^{* *}$ is the set of all partial continuous functions $f: \subseteq \Sigma^{*} \rightarrow \Sigma^{*}, F^{* \omega}$ is the set of all partial continuous functions $f: \subseteq \Sigma^{*} \rightarrow \Sigma^{\omega}, F^{\omega *}$ is the set of all partial continuous functions $f: \subseteq \Sigma^{\omega} \rightarrow \Sigma^{*}$ with open domain and $F^{\omega \omega}$ is the set of all partial continuous functions $f: \subseteq \Sigma^{\omega} \rightarrow \Sigma^{\omega}$ with $G_{\delta}$-domain (a $G_{\delta}$-set is a countable intersection of open sets). For $a, b \in\{*, \omega\}$, let $\eta^{a b}$ be the standard representations of $F^{a b}$ in [30, Def 2.3.10]. For representations 
$\gamma_{1}: \subseteq \Sigma^{a} \rightarrow M_{1}$ and $\gamma_{2}: \subseteq \Sigma^{b} \rightarrow M_{2}, a, b \in\{*, \omega\}$, a representation $\left[\gamma_{1} \rightarrow{ }_{p} \gamma_{2}\right]$ of the $\left(\gamma_{1}, \gamma_{2}\right)$-continuous functions $f: \subseteq M_{1} \rightarrow M_{2}$ is defined by $f=\left[\gamma_{1} \rightarrow p \gamma_{2}\right](q)$ $\Longleftrightarrow \eta_{q}^{a b}:=\eta^{a b}(q)$ realizes $f$ w.r.t. $\left(\gamma_{1}, \gamma_{2}\right)$ where The restriction of $\left[\gamma_{1} \rightarrow_{p} \gamma_{2}\right]$ to the total $\left(\gamma_{1}, \gamma_{2}\right)$-continuous functions is denoted by $\left[\gamma_{1} \rightarrow \gamma_{2}\right]$.

\subsection{Computable topological spaces}

Definition 2.1 (computable topological spaces). An effective topological space is a 4 -tuple $\mathbf{X}=(X, \tau, \beta, \nu)$ such that $(X, \tau)$ is a topological $T_{0}$-space and $\nu: \subseteq$ $\Sigma^{*} \rightarrow \beta$ is a notation of a base $\beta$ of $\tau$. $\mathbf{X}$ is a computable topological space if $\operatorname{dom}(\nu)$ is computable and

$$
\nu(u) \cap \nu(v)=\bigcup\{\nu(w):(u, v, w) \in S\} \text { for all } u, v \in \operatorname{dom}(\nu)
$$

for some c.e. set $S \subseteq(\operatorname{dom}(\nu))^{3}$.

Definition 2.2. Let $\mathbf{X}=(X, \tau, \beta, \nu)$ be an effective topological space.

Define a representation $\delta: \subseteq \Sigma^{\omega} \rightarrow X$ of the points as

$$
x=\delta(p) \Longleftrightarrow\left(\forall w \in \Sigma^{*}\right)(w \ll p \Longleftrightarrow x \in \nu(w))
$$

and a representation $\theta: \subseteq \Sigma^{\omega} \rightarrow \tau$ of the set of open sets as

$$
W=\theta(p) \Longleftrightarrow\left\{\begin{array}{l}
w \ll p \Rightarrow w \in \operatorname{dom}(\nu) \\
W=\bigcup\{\nu(w): w \ll p\} .
\end{array}\right.
$$

Define a representation $\delta^{-}: \subseteq \Sigma^{\omega} \rightarrow X$ of the points as

$$
\delta^{-}(p)=x \Longleftrightarrow \theta(p)=X \backslash \overline{\{x\}}
$$

where $\bar{A}$ is the closure of the set $A \subseteq X$ and define a representation $\psi^{-}: \subseteq$ $\Sigma^{\omega} \rightarrow \mathcal{A}$ of the set of closed sets as

$$
\psi^{-}(p)=X \backslash \theta(p) .
$$

A $\delta$-name of a point $x$ is a list of all names of all of its basic neighborhoods and a $\theta$-name of an open set $W$ is a list of base elements exhausting $W$.

Example 2.3 (computable topological spaces).

(i) (real line) Define $\mathbf{R}=(\mathbb{R}, \tau, \beta, \nu)$ such that $\tau$ is the real line topology and $\nu$ is a canonical notation of the set of all open intervals with rational endpoints. The representation $\delta$ for $\mathbf{R}$ is denoted by $\rho$.

(ii) (unit interval) Define $\mathbf{I}=\left([0,1], \tau^{\prime}, \beta^{\prime}, \nu^{\prime}\right)$ as the restriction of $\mathbf{R}$ to $[0,1]$. The representation $\delta$ for $\mathbf{I}$ is denoted by $\rho$. 
(iii) (extended real line with lower topology) Define $\overline{\mathbf{R}}_{<}^{+}=\left(\overline{\mathbb{R}}^{+}, \tau_{<}, \beta_{<}, \nu_{<}\right)$ where $\overline{\mathbb{R}}^{+}$is the set of non-negative reals and $+\infty, \tau_{<}$is the lower topology, $\beta_{<}$is the set

$$
\left\{\overline{\mathbb{R}}^{+}\right\} \cup\{(q,+\infty]: q \in \mathbb{Q} \cap[0,+\infty)\},
$$

and $\nu_{<}$is a canonical notation of $\beta_{<}$. The representation $\delta$ of points in $\overline{\mathbf{R}}_{<}^{+}$is denoted by $\bar{\rho}_{<}$.

(iv) (lower unit interval) Define $\mathbf{I}_{<}=\left([0,1], \tau_{I}^{\prime}, \beta_{<}^{\prime}, \nu_{<}^{\prime}\right)$ as the restriction of $\overline{\mathbf{R}}_{<}^{+}$to $[0,1]$.

We sometimes say that a point is computable to mean that the point is $\rho$ computable, that an open set is c.e. to mean that the open set is $\theta$-computable and that a closed set is co-c.e. to mean that the closed set is $\psi^{-}$-computable. On $\mathbb{R}$ or $\mathbb{I}$ we say that a real is c.e. to mean that the real is $\rho_{<- \text {computable and }}$ that a real $\alpha$ is right-c.e. if $D-\alpha$ is c.e. for some $D \in \mathbb{N}$. We also say that a function $f: \subseteq X \rightarrow X_{1}$ is computable to mean that $f$ is $\left(\delta, \delta_{1}\right)$-computable and that a function $f: X \rightarrow \overline{\mathbb{R}}^{+}$is lower semicomputable to mean that $f$ is $\left(\delta, \bar{\rho}_{<}\right)$-computable.

In what follows we assume that any effective topological space is equipped with these representations correspondingly. For example, for an effective topological space $\mathbf{X}_{1}=\left(X_{1}, \tau_{1}, \beta_{1}, \nu_{1}\right)$, the representation $\delta_{1}$ denotes the representation of points in $X_{1}$ defined here.

A variety of operations on points, sets and functions are computable w.r.t. the representations from Definition 2.2. We give some additional examples.

Theorem 2.4 ([33]).

(i) eval : $(f, x) \mapsto f(x)$ is $\left(\left[\delta_{1} \rightarrow_{p} \delta_{2}\right], \delta_{1}, \delta_{2}\right)$-computable.

(ii) For $f: \subseteq X_{1} \rightarrow X_{2}$ and $g: \subseteq X_{2} \rightarrow X_{3},(f, g) \mapsto g \circ f$ is $\left(\left[\delta_{1} \rightarrow_{p} \delta_{2}\right],\left[\delta_{2} \rightarrow_{p}\right.\right.$ $\left.\left.\delta_{3}\right],\left[\delta_{1} \rightarrow_{p} \delta_{3}\right]\right)$-computable.

(iii) There exists a $\left(\left[\delta_{1} \rightarrow_{p} \delta_{2}\right], \theta_{2}, \theta_{1}\right)$-computable function that maps every continuous function $f: \subseteq X_{1} \rightarrow X_{2}$ and every open set $W \subseteq X_{2}$ to some open set $T \subseteq X_{1}$ such that $f^{-1}[W]=T \cap \operatorname{dom}(f)$.

Weihrauch $[31,32]$ studied various computable versions of separation axioms. In this paper we use the following.

Definition 2.5 (axioms of computable separation). Let $\mathbf{X}$ be a computable topological space.

$\mathrm{CT}_{2}^{\prime}$ : There is a c.e. set $H \subseteq \Sigma^{*} \times \Sigma^{*}$ such that

$(\forall x, y, x \neq y)(\exists(u, v) \in H)(x \in \nu(u) \wedge y \in \nu(v))$ and

$(\forall(u, v) \in H)(\nu(u) \cap \nu(v)=\emptyset \vee(\exists x) \nu(u)=\{x\}=\nu(v))$.

$\mathrm{SCT}_{2}$ : There is a c.e. set $H \subseteq \Sigma^{*} \times \Sigma^{*}$ such that

$(\forall x, y, x \neq y)(\exists(u, v) \in H)(x \in \nu(u) \wedge y \in \nu(v))$ and $(\forall(u, v) \in H) \nu(u) \cap \nu(v)=\emptyset$. 
$\mathrm{SCT}_{3}$ : There are a c.e. set $R \subseteq \operatorname{dom}(\nu) \times \operatorname{dom}(\nu)$ and a computable function $r: \subseteq \Sigma^{*} \times \Sigma^{*} \rightarrow \Sigma^{\omega}$ such that for all $u, w \in \operatorname{dom}(\nu)$,

$\nu(w)=\bigcup\{\nu(u):(u, w) \in R\}$, $(u, w) \in R \Rightarrow \nu(u) \subseteq \psi^{-} \circ r(u, w) \subseteq \nu(w)$.

Weihrauch [32] also cosidered the axiom of $\mathrm{CT}_{2}$, which uses a multi-function in the definition. He showed $\mathrm{CT}_{2} \Longleftrightarrow \mathrm{CT}_{2}^{\prime}$. For simplicity, we prefer to call a space $\mathrm{CT}_{2}$ and to use the definition of $\mathrm{CT}_{2}^{\prime}$. Note that $\mathrm{SCT}_{3} \Rightarrow \mathrm{CT}_{2} \Rightarrow T_{2}$ and $\mathrm{SCT}_{3} \Rightarrow T_{3}$.

\section{Computability of measures}

In this section we study computability of measures via the representation approach.

In measure theory a lot of connections are known between the properties of $X$ and the corresponding properties of the spaces of measures such as completeness, compactness, metrizability and separability. Gács [8] proved that the space of measures on a computable metric space is another computable metric space. This result is another correspondence between a space and the space of measures on it.

In the result above the space of measures is equipped with the weak topology. On a more general space, however, A-topology is more natural. Recall that the A-topology coincides with the weak topology on a metric space. Similar connections are also known with the A-topology. Refer to [1, 8.10(iv)] for the detail. Here we show a version on a computable topological space of Gács' result.

\subsection{A computable topological space of measures}

Here we show that the space of measures on a computable topological space is another computable topological space with a natural structure. In what follows a measure is always a Borel measure.

Let $\mathbf{X}=(X, \tau, \beta, \nu)$ be a computable topological space. We consider the space $\mathcal{M}(X)$ of Borel measures. Let $\tau_{A}$ be the A-topology on $\mathcal{M}(X)$. The following sets form a countable subbase of the A-topology:

$$
\{\mu: \mu(G)>q\}
$$

where $G$ is a finite union of base sets and $q \in \mathbb{Q} \cap[0,1]$.

Then $\mathcal{M}(X)$ is second-countable because the above sets form a countable subbase. Furthermore the space $\mathcal{M}(X)$ is always $T_{0}$.

Proposition 3.1. The space $\mathcal{M}(X)$ with the A-topology is $T_{0}$.

Proof. Let $\mu_{1}, \mu_{2}$ be measures on $X$ such that $\mu_{1} \neq \mu_{2}$. Then there exists an open set $O$ such that $\mu_{1}(O) \neq \mu_{2}(O)$ by [1, Lemma 7.1.2.]. It follows that there exists a finite union $G$ of base sets such that $\mu_{1}(G) \neq \mu_{2}(G)$. We can assume 
that $\mu_{1}(G)<\mu_{2}(G)$ without loss of generality. Then there exists $q \in \mathbb{Q} \cap[0,1]$ such that $\mu_{1}(G)<q<\mu_{2}(G)$. Let $U=\{\mu: \mu(G)>q\}$. Note that $U$ is an open set on $\mathcal{M}(X)$ with the A-topology. We also have $\mu_{1} \notin U$ and $\mu_{2} \in U$. Hence the space is $T_{0}$.

Now we equip $\mathcal{M}(X)$ with a natural structure to be a computable topological space. For a notation $\mu: \subseteq \Sigma^{*} \rightarrow M$ define a notation of finite subsets as

$$
\mu^{\mathrm{fs}}(w)=W \Longleftrightarrow\left\{\begin{array}{l}
(\forall v \ll w) v \in \operatorname{dom}(\mu), \\
W=\{\mu(v): v \ll w\} .
\end{array}\right.
$$

Then $\nu^{\mathrm{fs}}$ is a notation of finite unions of base sets. Let $\mu_{A}$ be the notation of the countable subbase of the A-topology such that

$$
\mu_{A}(\langle u, v\rangle)=\left\{\mu: \mu\left(\nu^{\mathrm{fs}}(u)\right)>\nu_{\mathbb{Q} \cap[0,1]}(v)\right\} .
$$

Let $\nu_{A}=\bigcap \mu_{A}^{\mathrm{fs}}$. Then $\nu_{A}$ is a notation of a base. Let $\beta_{A}=\left\{\nu_{A}(w): w \in\right.$ $\left.\operatorname{dom}\left(\nu_{A}\right)\right\}$. Then $\beta_{A}$ is the base.

Proposition 3.2. The 4-tuple $\mathbf{M}(\mathbf{X})=\left(\mathcal{M}(X), \tau_{A}, \beta_{A}, \nu_{A}\right)$ is a computable topological space.

Proof. It is clear that $\mathbf{M}(\mathbf{X})$ is an effective topological space. All we have to do is to see whether (1) holds.

Let $\sigma_{i} \in \operatorname{dom}\left(\nu_{A}\right)$ for $i=1,2$. Then there exists finite sets $W_{i}$ such that $W_{i}=\left\{\tau_{i}: \tau_{i} \ll \sigma_{i}\right\}$. Note that $\tau_{i} \in W_{i} \Rightarrow \tau_{i} \in \operatorname{dom}\left(\mu_{A}\right)$. Let $W=W_{1} \cup W_{2}$. Then

$$
\bigcap_{\tau \in W} \mu_{A}(\tau)=\bigcap_{\tau_{1} \in W_{1}} \mu_{A}(\tau) \cap \bigcap_{\tau_{2} \in W_{2}} \mu_{A}(\tau)=\nu_{A}\left(\sigma_{1}\right) \cap \nu_{A}\left(\sigma_{2}\right) .
$$

Hence one can computably find $\sigma_{3}$ from $\sigma_{1}$ and $\sigma_{2}$ such that $\nu_{A}\left(\sigma_{1}\right) \cap \nu_{A}\left(\sigma_{2}\right)=$ $\nu_{A}\left(\sigma_{3}\right)$. This ensures the existence of a c.e. set $S_{A} \subseteq\left(\left(\Sigma^{*}\right)^{3}\right)$ satisfying (1).

We give an intuitive interpretation of $\delta_{A}$-name $p$ of $\mu \in \mathbf{M}$. For each finite union $G$ of base sets, we can enumerate all rationals $q$ such that $\mu(G)>q$ from $p$. We say that $\mu(G)$ is approximated from below by $p$. Conversely suppose that $\mu(G)$ is approximated from below for each $G$ with help from $p^{\prime}$. Then we can construct a representation of $\mu$ from $p^{\prime}$. In the following we use this argument without giving further details. By this discussion, it is easy to see that $\delta_{A}$ is equivalent to the canonical representation in Schröder [23].

Now the following definition is natural. Note that this definition is a general version of that of a computable measure on a computable metric space in $[8,11]$.

Definition 3.3. Let $\mathbf{X}=(X, \tau, \beta, \nu)$ be a computable topological space. $A$ measure on $X$ is computable if it is a computable point on the computable topological space $\mathbf{M}$.

The following is immediate.

Proposition 3.4. A measure $\mu$ is computable iff the meausre of the finite union of base sets is uniformly approximated from below. 


\subsection{Some properties}

From here we study some computable operations on $\mathbf{M}$.

Proposition 3.5. Let $\mathbb{R}^{+}$be the set of non-negative reals and $\overline{\mathbb{R}}^{+}=\mathbb{R}^{+} \cup\{\infty\}$. We also write $\mathcal{M}$ to mean $\mathcal{M}(X)$.

(i) The plus operation $+: \mathcal{M} \times \mathcal{M} \rightarrow \mathcal{M}$ is $\left(\delta_{A}, \delta_{A}, \delta_{A}\right)$-computable.

(ii) Let eval : $\mathcal{M} \times \tau \rightarrow \mathbb{I}$ be such that eval $(\mu, G)=\mu(G)$. Then eval is $\left(\delta_{A}, \theta, \rho_{<}\right)$-computable.

(iii) The integral operation $\int: C\left(X, \mathbb{R}^{+}\right) \times \mathcal{M} \rightarrow \overline{\mathbb{R}}^{+}$is $\left(\left[\delta \rightarrow \rho_{<}\right], \delta_{A}, \bar{\rho}_{<}\right)$computable.

(iv) The integral operation $\int: C_{b}\left(X, \mathbb{R}^{+}\right) \times \mathcal{M} \rightarrow \mathbb{R}^{+}$is $\left([\delta \rightarrow \rho], \delta_{A}, \rho\right)$ computable.

Proof. (i) Let $\mu_{1}, \mu_{2}$ be measures in $\mathcal{M}(X)$ and $p_{1}, p_{2}$ be $\delta_{A}$-names respectively. For each finite union $G$ of base sets, $\mu_{1}(G)$ and $\mu_{2}(G)$ are approximated from below by $p_{1}$ and $p_{2}$. Hence $\left(\mu_{1}+\mu_{2}\right)(G)$ is also approximated from below by $p_{1}$ and $p_{2}$. Then one can construct a name of $\mu_{1}+\mu_{2}$ from $p_{1}$ and $p_{2}$.

(ii) For each open set $G$, we can enumerate all finite union of base sets which is contained in $G$ by a $\theta$-name of $G$. The measure $\mu$ of each finite union of base sets is approximated by rationals from below by a $\delta_{A}$-name of $\mu$. This follows that $\mu(G)$ is approximated by rationals from below.

(iii) Let $f \in \mathcal{C}\left(X, \mathbb{R}^{+}\right)$and $\mu \in \mathcal{M}(X)$. Note that

$$
\int f d \mu=\sup \left\{\sum_{i=1}^{k}\left(a_{i}-a_{i-1}\right) \cdot \mu\left(f^{-1}\left(a_{i}, \infty\right)\right): 0=a_{0}<a_{1}<\ldots<a_{k}, a_{i} \in \mathbb{Q}\right\} .
$$

Since $\left(a_{i}, \infty\right)$ is open in $\mathbb{R}^{+}$for each $i$, one obtains $\theta$-name of $f^{-1}\left(a_{i}, \infty\right)$ by the representation of $f$ by Proposition 2.4. Hence $\mu\left(f^{-1}\left(a_{i}, \infty\right)\right)$ is approximated by rationals from below by the representations of $f$ and $\mu$ by (ii).

(iv) Let $f \in \mathcal{C}_{b}\left(X, \mathbb{R}^{+}\right)$and $\mu \in \mathcal{M}(X)$. Since $f$ is bounded, there exists $D$ such that $D-f \in \mathcal{C}\left(X, \mathbb{R}^{+}\right)$. Then

$$
\int_{X} f d \mu=D \mu(X)-\int_{X}(D-f) d \mu(<\infty)
$$

is approximated by rationals from above.

\section{Universality}

Here we study the existence of a universal Martin-Löf test on a computable topological space with a computable measure. Zvonkin and Levin [36] showed 
the existence of a universal ML-test with a measure such that the measure of the finite unions of base sets is uniformly computable. Hertling and Weihrauch [10] showed the existence with a measure such that the measure of the finite unions of base sets is uniformly right-c.e. From now on we consider a computable measure in our definition, that is, a measure such that the measure of the finite unions of base sets is uniformly c.e.

\subsection{Definition}

Let $\mathbf{X}=(X, \tau, \beta, \nu)$ be a computable topological space and $\mu$ be a a computable non-negative probabilistic Borel measure.

Definition 4.1 ([36, 10]). A Martin-Löf test (or ML-test) on $\mathbf{X}$ is a sequence of uniformly c.e. open sets $\left\{U_{n}\right\}$ with $\mu\left(U_{n}\right) \leq 2^{-n}$. A point $x \in X$ is Martin-Löf random (or ML-random) if it passes all ML-tests, that is, $x \notin \bigcap_{n} U_{n}$.

Definition 4.2 (universal test). A ML-test $\left\{U_{n}\right\}$ is universal if, for each $M L$ test $\left\{V_{n}\right\}$, we have $\bigcap_{n} V_{n} \subseteq \bigcap_{n} U_{n}$.

The question of this section is whether a universal test exists. It should be noted that the existence of a universal test is equivalent to the existsnce of a universal integral test.

Definition 4.3. An integral test is a lower semicomputable function $t: X \rightarrow$ $\overline{\mathbb{R}}^{+}$such that $\int t d \mu \leq 1$. An integral test is universal if, for each integral test $f$, we have $\{x \mid f(x)=\infty\} \subseteq\{x \mid t(x)=\infty\}$.

Proposition 4.4. A point $x \in X$ is $M L$-random iff $t(x)<\infty$ for all integral tests.

Proof. Suppose that $x$ is not ML-random. Then there exists a ML-test $\left\{U_{n}\right\}$ with $x \in \bigcap_{n} U_{n}$. We can assume that $\left\{U_{n}\right\}$ is decreasing. Let $t(y)=\sup _{n}\{n \mid$ $\left.y \in U_{n}\right\}$. Then $t$ is lower semicomputable and $\int t d \mu<\infty$. Hence $t^{\prime}=t / c$ is an integral test for some $c \in \mathbb{N}$. Since $\bigcap_{n} U_{n}=\{x \mid t(x)=\infty\}$, we have $t^{\prime}(x)=\infty$.

Suppose that there exists an integral test $t$ such that $t(x)=\infty$. Let $U_{n}=$ $\left\{y \mid t(y)>2^{n}\right\}$. Then $\left\{U_{n}\right\}$ is a ML-test. Since $\bigcap_{n} U_{n}=\{x \mid t(x)=\infty\}$, we have $x \in \bigcap_{n} U_{n}$.

Proposition 4.5. There exists a universal $M L$-test on $\mathbf{X}$ with $\mu$ iff there exists a universal integral test on it.

Proof. Suppose that there exists a universal test $\left\{U_{n}\right\}$. Let $t$ be the integral test such that $\bigcap_{n} U_{n}=\{x \mid t(x)=\infty\}$ as we saw in the proof of Proposition 4.4. Let $f$ be an arbitrary integral test. Then there exists a ML-test $\left\{V_{n}\right\}$ such that $\bigcap_{n} V_{n}=\{x \mid f(x)=\infty\}$. Since $\left\{U_{n}\right\}$ is universal, $t$ is also universal. The other direction is proved in the same manner. 


\subsection{When a universal test exists}

It should be noted that, on a computable metric space with a computable measure, a universal test always exists.

Theorem 4.6 (Hoyrup and Rojas [11], partially by Gács [8]). There exists a universal test on a computable metric space with a computable measure.

We also know that $\mathrm{SCT}_{3}$ is a sufficient condition for computable metrization.

Theorem 4.7 ([22, 9, 28]). Suppose that $\mathbf{X}$ is $\mathrm{SCT}_{3}$. Then its topology is generated by some computable metric.

Then we can conclude that $\mathrm{SCT}_{3}$ is a sufficient condition for the existence of a universal test.

Theorem 4.8. Suppose that $\mathbf{X}$ is $\mathrm{SCT}_{3}$. Then there exists a universal test on $\mathbf{X}$ with a computable measure $\mu$.

Here we give a direct proof of this theorem to see how the regularity is used. We prepare some lemmas. We write $U_{n} \uparrow U$ to mean that $U_{n} \subseteq U_{n+1}$ for all $n$ and $\lim _{n} U_{n}=U$.

Lemma 4.9. Suppose that $\mathbf{X}$ is $\mathrm{SCT}_{3}$. For each c.e. open set $W$, one can construct a sequence $\left\{U_{n}\right\}$ of uniformly c.e. open sets and a sequencce $\left\{V_{n}\right\}$ of uniformly co-c.e. closed sets such that

(i) $U_{n} \uparrow W, V_{n} \uparrow W$,

(ii) $U_{n} \subseteq V_{n} \subseteq W$ for all $n$.

Further $U_{n}$ can be the finite union of base sets for each $n$.

Proof. First we assume that $W$ is a base set. Let $R$ and $r$ be in the definition of $\operatorname{SCT}_{3}$. Let $w \in \operatorname{dom}(\nu)$ be such that $\nu(w)=W$. Since $R$ is c.e., one can construct a computable sequence $\left\{u_{n}\right\}$ in $\Sigma^{*}$ such that

$$
\nu(w)=\bigcup\{\nu(u) \mid(u, w) \in R\}=\bigcup_{n}\left\{u_{n} \mid n \in \mathbb{N}\right\} .
$$

Let $U_{n}=\bigcup_{i \leq n} u_{i}$ and $V_{n}=\bigcup_{i \leq n} \psi^{-} \circ r\left(u_{i}, w_{i}\right)$. Then $U_{n} \uparrow W$ and $V_{n} \uparrow W$. By the property of $r$ we have $\nu\left(u_{i}\right) \subseteq \psi^{-} \circ r\left(u_{i}, w\right) \subseteq \nu(w)$. Then $U_{n} \subseteq V_{n} \subseteq W$.

Next we prove the lemma in a general case. Since $W$ is c.e. open, one can construct a computable sequence $\left\{W_{m}\right\}$ of base sets such that

$$
W=\bigcup_{m} W_{m}
$$

Then one can construct a computable double sequence $\left\{U_{n}^{m}\right\}$ of open sets and a computable double sequence $\left\{V_{n}^{m}\right\}$ of closed sets such that $U_{n}^{m} \uparrow W^{m}, V_{n}^{m} \uparrow$ $W^{m}$ for each $m$ and $U_{n}^{m} \subseteq V_{n}^{m} \subseteq W^{m}$ for each $n, m$. Let

$$
\widehat{U}_{k}=\bigcup_{i \leq k} U_{k}^{i} \text { and } \widehat{V}_{k}=\bigcup_{i \leq k} V_{k}^{i}
$$


for all $k$. Then $\left\{\widehat{U}_{k}\right\}$ is a computable sequence of finite unions of base sets and $\left\{\widehat{V}_{k}\right\}$ is a sequence of uniformly co-c.e. closed sets. By the construction, $\widehat{U}_{k} \subseteq \widehat{V}_{k} \subseteq W$ for all $k$.

We show that $\widehat{U}_{k} \uparrow W$. Note that

$$
\widehat{U}_{k}=\bigcup_{i \leq k} U_{k}^{i} \subseteq \bigcup_{i \leq k+1} U_{k+1}^{i}=\widehat{U}_{k+1} .
$$

Let $x \in W$. Then there exists $m$ such that $x \in W_{m}$. Since $U_{n}^{m} \uparrow W_{m}$, there exists $n$ such that $x \in U_{n}^{m}$. Let $k=\max \{m, n\}$. Then

$$
x \in U_{k}^{k} \subseteq \bigcup_{i \leq k} U_{k}^{i}=\widehat{U}_{k} .
$$

Hence $W \subseteq \bigcup_{k} \widehat{U}_{k}$. It follows that $\widehat{U}_{k} \uparrow W$.

Finally we show that $\widehat{V}_{k} \uparrow W$. This is because $\widehat{V}_{k}$ is increasing and $\widehat{U}_{k} \subseteq$ $\widehat{V}_{k} \subseteq W$ for all $k$.

Proof of Theorem 4.8. Let $k \in \mathbb{N}$. First we construct a c.e. open set $\widetilde{W}$ from $W$ such that

(i) $\mu(\widetilde{W}) \leq 2^{-k}$,

(ii) $\mu(W) \leq 2^{-k}$ implies $\widetilde{W}=W$. $\widetilde{W}$ as

For each c.e. open set $W$, construct $\left\{U_{n}\right\}$ and $\left\{V_{n}\right\}$ as Lemma 4.9. We define

$$
\widetilde{W}=\bigcup_{n}\left\{U_{n} \mid \mu\left(V_{n}\right) \leq 2^{-k}\right\} .
$$

Since $\mu$ is computable and $V_{n}$ is co-c.e. closed set, $\mu\left(V_{n}\right)$ is right-c.e. Hence $\widetilde{W}$ is c.e. open.

We show $\mu(\widetilde{W}) \leq 2^{-k}$. If $\mu\left(V_{n}\right) \leq 2^{-k}$, then

$$
\mu\left(\bigcup_{i \leq n} U_{i}\right)=\mu\left(U_{n}\right) \leq \mu\left(V_{n}\right) \leq 2^{-k} .
$$

Suppose that $\mu\left(V_{n_{0}}\right)>2^{-k}$ and $\mu\left(V_{n_{0}-1}\right) \leq 2^{-k}$ for some $n_{0}$. Then $\mu\left(V_{n}\right) \geq$ $\mu\left(V_{n_{0}}\right)>2^{-k}$ for all $n \geq n_{0}$. It follows that $\mu(\widetilde{W}) \leq \mu\left(\bigcup_{i \leq n_{0}} U_{i}\right) \leq 2^{-k}$. Suppose that $\mu\left(V_{n}\right) \leq 2^{-k}$ for all $n$. Then $\mu(W)=\mu\left(\bigcup_{n} U_{n}\right)=\sup _{n} \mu\left(U_{n}\right) \leq$ $2^{-k}$.

If $\mu(W) \leq 2^{-k}$, then $\mu\left(V_{n}\right) \leq 2^{-k}$ for all $n$ and $\widetilde{W}=\bigcup_{n} U_{n}=W$.

Since one can computably enumerate all sequences of uniformly c.e. open sets, there exists a double sequence $\left\{W_{n}^{m}\right\}$ of uniformly c.e. open sets satisfying the following: if $\left\{A_{n}\right\}$ be a sequence of uniformly c.e. open sets, then there exists $m$ such that $A_{n}=W_{n}^{m}$ for all $n$. For each $m, n$, let $\widetilde{W}_{n}^{m}$ be the c.e. open set such that 
(i) $\mu\left(\widetilde{W}_{n}^{m}\right) \leq 2^{-n}$,

(ii) $\mu\left(\widetilde{W}_{n}^{m}\right) \leq 2^{-n}$ impliew $\widetilde{W}_{n}^{m}=W_{n}^{m}$.

Then $\left\{\left\{\widetilde{W}_{n}^{m}\right\}_{n}\right\}_{m}$ is a computable enumeration of all ML-random tests.

Let $T_{n}=\bigcup_{i} \widetilde{W}_{n+i+1}^{i}$. Then $\left\{T_{n}\right\}$ is a sequence of uniformly c.e. oepn sets. Further

$$
\mu\left(T_{n}\right) \leq \sum_{i} \mu\left(\widetilde{W}_{n+i+1}^{i}\right) \leq \sum_{i} 2^{-n-i-1}=2^{-n} .
$$

Hence $\left\{T_{n}\right\}$ is a ML-test. For any ML-test $\left\{A_{n}\right\}$, there exists $m$ such that $A_{n}=\widetilde{W}_{n}^{m}$ for all $n$. Then $A_{n+m+1} \subseteq \widetilde{W}_{n+m+1}^{m} \subseteq T_{n}$ for all $n$. Hence $\left\{T_{n}\right\}$ is universal.

\subsection{A universal test does not exist in general}

Here we give negative results. First it should be noted that there does not exist a universal ML-test in general. Although we give a stronger result later, the following example is easy to understand and is worth noting.

Proposition 4.10. There exists a computable topological space $\mathbf{X}$ and a computable measure $\mu$ on it such that no test is universal.

Proof. We consider the lower unit interval $\mathbf{I}_{<}$in Example 2.3. Let $\alpha$ be a noncomputable c.e. real with $\alpha<1$. Let $\mu$ be the Dirac measure at $\alpha$. In other words the measure $\mu$ satisfies the following:

$$
\mu(U)=\left\{\begin{array}{l}
1 \text { if } \alpha \in U \\
0 \text { otherwise }
\end{array}\right.
$$

We show that $\mu$ is computable. For each rational $q<1$, the relation $q<\alpha$ is semidecidable. Hence $\mu((q, 1])$ is uniformly c.e. Since each finite union of base sets has the form $(q, 1], \mu$ is computable.

Next we show that the set of non-ML-random points on $\mathbf{I}_{<}$with $\mu$ is

$$
\{x \mid \alpha<x \leq 1\} .
$$

Let $x$ be a point such that $\alpha<x \leq 1$. Then there exists a rational $q$ such that $\alpha<q<x$. Let $U_{n}=(q, 1]$ for all $n$. Since $\mu\left(U_{n}\right)=0$ for all $n,\left\{U_{n}\right\}$ is a ML-test. Further $x \in(q, 1]=\bigcap_{n} U_{n}$. Then $x$ is not ML-random.

Let $x$ be a point such that $0 \leq x \leq \alpha$. Suppose that $x$ is not ML-random for a contradiction. Then there exists a ML-test $\left\{V_{n}\right\}$ with $x \in \bigcap_{n} V_{n}$. Since $x \in V_{1}, \alpha \in V_{1}$ and $\mu\left(V_{1}\right)=1$. This is a contradiction. Hence $x$ is ML-random.

Let $\left\{W_{n}\right\}$ be a ML-test. Suppose that $\bigcap_{n} W_{n}=\{x \mid \alpha<x \leq 1\}$. Since $W_{1}$ is c.e. open, there exists a right-c.e. real $\beta$ such that $W_{1}=(\beta, 1]$. Since $\bigcap_{n} W_{n} \subseteq W_{1}$, we have $\beta \leq \alpha$. If $\beta<\alpha$, then $\mu\left(W_{1}\right)=\mu((\beta, 1])=1>2^{-1}$. Hence $\beta=\alpha$. Since $\alpha$ is lower semicomputable and $\beta$ is right-c.e., $\alpha$ is a computable real. This is a contradiction. Hence $\left\{W_{n}\right\}$ is not universal. Since $\left\{W_{n}\right\}$ is arbitrary, there does not exist a universal ML-test. 


\subsection{When a universal test does not exist}

We knew that $\mathrm{SCT}_{3}$ is a sufficient condition for the existence of a universal test. How much do we weaken the condition. We show that $\mathrm{CT}_{2}$ is not sufficient.

Theorem 4.11. There exists a $\mathrm{CT}_{2}$ space $\mathbf{X}$ with a computable measure $\mu$ such that no universal test exists.

Proof. Let $A \subseteq \mathbb{N}$ be a non-computable c.e. set such that $0 \in A$. Consider the space $X=\{0\} \cup \mathbb{N} \backslash A$ with the discrete topology $\tau$. We define a base $\beta$ of $\tau$ as

$$
\beta=\{\{n\}: n \in X\}
$$

and a notation $\nu: \subseteq \Sigma^{*} \rightarrow \beta$ of the base $\beta$ as

$$
\nu(\bar{i})= \begin{cases}\{0\} & \text { if } i \in A \\ \{i\} & \text { if } i \notin A\end{cases}
$$

where $\bar{i}$ is the binary representation of $i$.

We show that $\mathbf{X}=(X, \tau, \beta, \nu)$ is a computable topological space. Clearly, $\operatorname{dom}(\nu)$ is computable. We define a c.e. set $S \subseteq(\operatorname{dom}(\nu))^{3}$ as follows. If $i, j \in A$, then $(\bar{i}, \bar{j}, w) \in S \Longleftrightarrow w=\bar{i}$. If $i \notin A$ or $j \notin A$, then $(\bar{i}, \bar{j}, w) \in S \Longleftrightarrow w=$ $\bar{i}=\bar{j}$. It is not difficult to see that $S$ satisfies (1) in Definitoin 2.1.

We show that $\mathbf{X}$ is $\mathrm{CT}_{2}$. We define a c.e. set $H \subseteq \Sigma^{*} \times \Sigma^{*}$ as

$$
H=\{(\bar{i}, \bar{j}): i \neq j\} \text {. }
$$

We prove that $H$ satisfies the property in the definition of $\mathrm{CT}_{2}^{\prime}$. Let $(\bar{i}, \bar{j}) \in H$. If $i, j \in A$, then $\nu(\bar{i})=\nu(\bar{j})=\{0\}$. If $i \notin A$ or $j \notin A$, then $\nu(\bar{i}) \cap \nu(\bar{j})=\emptyset$ because $i \neq j$. Let $i, j \in X$ such that $i \neq j$. Then $i \in \nu(\bar{i})$ and $j \in \nu(\bar{j})$.

We define a measure $\mu$ as

$$
\mu(\{0\})=1 .
$$

We show that $\mu$ is computable. Let $\overline{a_{1}}, \ldots, \overline{a_{k}} \in \operatorname{dom}(\nu)$. Note that

$$
\mu\left(\nu\left(\overline{a_{1}}\right) \cup \ldots \cup \nu\left(\overline{a_{k}}\right)\right)= \begin{cases}1 & \text { if } a_{j} \in A \text { for some } j \leq k \\ 0 & \text { otherwise. }\end{cases}
$$

Then the measure is uniformly lower semicomputable. Hence $\mu$ is computable.

We show that the set of non-ML-random points is $\mathbb{N} \backslash A$. Since $\mu(\{0\})=1$, the natural number 0 is ML-random. For each $i \notin A$, let $U_{n}=\{\bar{i}\}$ for all $n$. Then $\left\{U_{n}\right\}$ is a sequence of uniformly c.e. open sets with $\mu\left(U_{n}\right)=0$. Hence $\left\{U_{n}\right\}$ is a ML-test. It follows that the natural number $i$ is not ML-random.

We claim that $\mathbb{N} \backslash A$ is open but not c.e. Suppose that $\mathbb{N} \backslash A$ is c.e. Then there exists a computable sequence $\left\{a_{n}\right\}$ of natural numbers such that $\mathbb{N} \backslash A=$ $\bigcup_{n} \nu\left(\overline{a_{n}}\right)$. If $a_{n} \in A$, then $\{0\}=\nu\left(\overline{a_{n}}\right) \subseteq \mathbb{N} \backslash A$, which is a contradiction. Hence $a_{n} \notin A$ for all $n$. It follows that

$$
\left\{a_{n}: n \in \mathbb{N}\right\}=\bigcup_{n} \nu\left(\overline{a_{n}}\right)=\mathbb{N} \backslash A .
$$


Since the complement of $A$ is c.e. and $A$ is c.e. by the definition, $A$ is computable, which is a contradiction.

We prove that each ML-random test is not universal. Let $\left\{V_{n}\right\}$ be a ML-test. Since $\mu\left(V_{1}\right) \leq 2^{-1}, 0 \notin V_{1}$. Hence $V_{1} \subseteq \mathbb{N} \backslash A$. Since $\mathbb{N} \backslash A$ is not c.e., we have $V_{1} \subsetneq \mathbb{N} \backslash A$. Hence $\left\{V_{n}\right\}$ is not universal.

It should be noted that $\mathbf{X}$ constructed above is not $\mathrm{SCT}_{3}$ by Theorem 4.8 . The space $\mathbf{X}$ is not even $\mathrm{SCT}_{2}$. Suppose that $\mathbf{X}$ is $\mathrm{SCT}_{2}$ and $H$ is in the definition of $\mathrm{SCT}_{2}$. We define a c.e. set $S \subseteq \mathbb{N}$ as

$$
S=\left\{j:\left(0^{i} 1,0^{j} 1\right) \in H \text { and } i \in A\right\} \cup\left\{i:\left(0^{i} 1,0^{j} 1\right) \in H \text { and } j \in A\right\} .
$$

We show that $S \subseteq \mathbb{N} \backslash A$. Consider $j \in S, i \in A$ and $\left(0^{i} 1,0^{j} 1\right) \in H$. Then $\mu\left(0^{i} 1\right)=\{0\}$ and $\nu\left(0^{i} 1\right) \cap \mu\left(0^{j} 1\right)=\emptyset$. Hence $j \notin A$. It follows that $S \subseteq \mathbb{N} \backslash A$.

We show that $S \supseteq \mathbb{N} \backslash A$. For each $i \in \mathbb{N} \backslash A$, there exists $(u, v) \in H$ such that $i \in \nu(u)$ and $0 \in \nu(v)$. Since $i \neq 0, u=0^{i} 1$. Since $0 \in \nu(v), v=0^{j} 1$ and $j \in A$ for some $j$. Then $i \in S$.

However $S=\mathbb{N} \backslash A$ is impossible, since $S$ is c.e. and $\mathbb{N} \backslash A$ is not c.e. Hence $\mathbf{X}$ is not $\mathrm{SCT}_{2}$.

The author does not know whether there exists an $\mathrm{SCT}_{2}$ space with a computable measure on it such that there does not exist a universal ML-test.

\section{Complexity randomness}

Martin-Löf randomness on Cantor space has a characterization by complexity. In this section we study whether Martin-Löf randomness on a computable topological space with a computable measure has a characterization by complexity.

\subsection{Definition}

On Cantor space with a computable measure $\mu$, a binary sequence $Z \in 2^{\omega}$ is not Martin-Löf random iff for all $d \in \mathbb{N}$ there exists $n$ such that

$$
K(Z\lceil n)<-\log \mu(\llbracket Z\lceil n \rrbracket)-d
$$

where $K$ is the universal prefix-free Kolmogorov complexity, $Z\lceil n$ is the first $n$ bits of the sequence $Z, \log (0)=-\infty, \llbracket \sigma \rrbracket=\left\{X \in 2^{\omega} \mid \sigma \prec X\right\}$ and $\prec$ is the prefix relation. To prove the characterization by complexity, it is important that the relation (2) is semidecidable, which is based on the fact that $\mu(\llbracket \sigma \rrbracket)$ is uniformly computable, more precisely, uniformly right-c.e.

On a computable metric space with a computable measure, Hoyrup and Rojas [11] have given a characterization by complexity of Martin-Löf randomness. They devide the whole space into cells whose measures are uniformly computable. However we can not generalize it to a computable topological space in the same manner. 
We wish to have a characterization like this: on a computable topological space $\mathbf{X}$ with a computable measure $\mu$, a point $x \in X$ is not Martin-Löf random iff for all $d \in \mathbb{N}$ there exists some set $A$ such that

$$
x \in A \text { and } H(A)<-\log \mu(A)-d
$$

where $H$ is some kind of complexity. We require the set $A$ to be co-c.e. closed so that $\mu(A)$ is right-c.e. Then the set $A$ can be identified with a computable $\psi^{-}$-name $p$. We use the monotone complexity $K m$ so that $H(A)=K m(A)$ can be finite.

A monotone machine is a Turing machine, which reads finite strings from an input tape, operates on work taps and write one-way finite strings or infinite sequences to an output tape. Then a monotone machine is similar to a Type-2 machine. We write $M(\sigma) \downarrow$ if $M$ reads exactly $\sigma$ from its input tape and write some finite string or some infinite sequences to its output tape. Let $M(\sigma)$ denote the string or the sequence.

Another way to define a monotone machine is that a c.e. set of pairs $(\sigma, \tau)$ where $\sigma, \tau \in 2^{*}$ and for every pair $\left(\sigma_{1}, \tau_{1}\right),\left(\sigma_{2}, \tau_{2}\right) \in M, \sigma_{1} \preceq \sigma_{2}$ implies $\tau_{1} \preceq \tau_{2}$ or $\tau_{2} \preceq \tau_{1}$.

Definition 5.1 (Monotone complexity; Levin $[13,14])$. We define the monotone complexity of $\tau \in 2^{*} \cup 2^{\omega}$ with respect to $M$ to be

$$
K m^{M}(\tau)=\min \{|\sigma|: \tau \preceq M(\sigma) \downarrow\}
$$

or equivalently

$$
K m^{M}(\tau)=\min \{|\sigma|:(\sigma, \rho) \in M \text { for some } \rho \succeq \tau\}
$$

if $\tau \in 2^{*}$ and

$$
K m^{M}(\tau)=\sup _{n} K m(\tau\lceil n)
$$

if $\tau \in 2^{\omega}$. One can show that there is a universal monotone machine $U$ and we define $K m(\tau)=K m^{U}(\tau)$.

The following are well-known results in the theory of algorithmic randomness $[6,18]$.

Proposition 5.2. The function $K m$ is monotone, that is, $\sigma \preceq \tau$ implies $K m(\sigma) \leq K m(\tau)$ for all $\sigma, \tau \in 2^{*} \cup 2^{\omega}$.

Proposition 5.3. A sequence $Z \in 2^{\omega}$ is computable iff there exists $d \in \mathbb{N}$ such that $\operatorname{Km}(Z)<d$.

Theorem 5.4. A sequence $Z \in 2^{\omega}$ is Martin-Löf random iff there exists $d$ such that, for all $n, K m(Z\lceil n)>n-d$.

The following fact is a simple important property of monotone machines.

Proposition 5.5. Let $M, N$ be monotone machines. Then $M \circ N$ is also a monotone machine. 
Now we give the definition of complexity randomness. We assume that $\Sigma=\{0,1\}=2$ without loss of generality so that each $\psi^{-}$-name $p$ of a closed set is in $\{0,1\}^{\omega}=2^{\omega}$. For a closed set $A$, we define

$$
K m(A)=\min _{p}\left\{K m(p) \mid \psi^{-}(p)=A\right\} .
$$

It should be noted that $\operatorname{Km}(A)$ is finite iff $A$ is co-c.e. closed, because if $A$ is not co-c.e. closed, then each $\psi^{-}(p)=A$ implies $K m(p)=\infty$.

Definition 5.6. Let $\mathbf{X}=(X, \tau, \beta, \nu)$ be a computable topological space and $\mu$ be a computable measure on it. A point $x \in X$ is complexity random if there exists $d \in \mathbb{N}$ such that

$$
x \in A \Rightarrow K m(A) \geq-\log \mu(A)-d
$$

for each closed set $A$. Here we define $\log (0)=-\infty$.

\subsection{Independence from the notation}

The monotone complexity $K m$ depends on the representation $\psi^{-}$, which also depends on the notation $\nu$. However we can replace the notation with an equivalent one.

Definition 5.7 ([33]). The computable topological spaces $\mathbf{X}_{1}=\left(X, \tau, \beta_{1}, \nu_{1}\right)$ and $\mathbf{X}_{2}=\left(X, \tau, \beta_{2}, \nu_{2}\right)$ are equivalent iff $\nu_{1} \leq \theta_{2}$ and $\nu_{2} \leq \theta_{1}$.

Theorem 5.8 (robustness [33]). Let $\mathbf{X}_{1}=\left(X, \tau, \beta_{1}, \nu_{1}\right)$ and $\mathbf{X}_{2}=\left(X, \tau, \beta_{2}, \nu_{2}\right)$ be computable topological spaces. Then $\mathbf{X}_{1}$ and $\mathbf{X}_{2}$ are equivalent $\Longleftrightarrow \theta_{1} \equiv \theta_{2}$.

Proposition 5.9. Let $\mathbf{X}_{1}=\left(X, \tau, \beta_{1}, \nu_{1}\right)$ and $\mathbf{X}_{2}=\left(X, \tau, \beta_{2}, \nu_{2}\right)$ be computable topological spaces. Let $K m_{1}$ and $K m_{2}$ be the monotone complexities of closed sets on $\mathbf{X}$ and $\mathbf{X}^{\prime}$ respectively. If $\theta_{2} \leq \theta_{1}$, then there exists $d \in \mathbb{N}$ such that

$$
K m_{1}(A) \leq K m_{2}(A)+d
$$

for all closed sets $A$.

Proof. Since $\theta_{2} \leq \theta_{1}$, there exists a computable function $h: \subseteq 2^{\omega} \rightarrow 2^{\omega}$ such that $\theta_{2}(p)=\theta_{1} \circ h(p)$ for all $p \in \operatorname{dom}\left(\theta_{1}\right)$. Further $h$ can be seen as a monotone machine, more precisely, there exists a monotone machine $N$ satisfying the following: for each $n$ and $p \in \operatorname{dom}\left(\theta_{1}\right)$, there exists $(\sigma, \tau) \in N$ such that $\sigma \prec p$ and $h(p)\lceil n \prec \tau$. Consider the monotone machine $N \circ M$. By universality of $M$, there exists $d$ such that $K m(\sigma) \leq K m^{N \circ M}(\sigma)+d$ for all $\sigma \in 2^{*} \cup 2^{\omega}$.

For each co-c.e. closed set $A$, let $p \in 2^{\omega}$ be a computable sequence such that $K m_{2}(A)=K m(p)$ and $\psi_{2}^{-}(p)=A$. Further let $\sigma \in 2^{*}$ such that $K m(p)=|\sigma|$ and $p \preceq M(\sigma)$. Then $h(p) \preceq N \circ M(\sigma)$. Hence $K m^{N \circ M}(h(p)) \leq|\sigma|$. It follows that $K m(\sigma) \leq|\sigma|+d$ and

$$
K m_{1}(A) \leq|\sigma|+d=K m(p)+d=K m_{2}(A)+d .
$$

Finally note that $K m_{1}(A)=K m_{2}(A)=\infty$ if $A$ is not co-c.e. closed. 
Corollary 5.10. Let $\mathbf{X}_{1}=\left(X, \tau, \beta_{1}, \nu_{1}\right)$ and $\mathbf{X}_{2}=\left(X, \tau, \beta_{2}, \nu_{2}\right)$ be equivalent computable topological spaces and $\mu$ be a computable measure on it. Then complexity randomness on the spaces coincide.

It should be noted that the computability of a measure $\mu$ does not depend on the notation.

\subsection{Some natural properties}

Here we rephrase the definition of complexity randomness in some forms and show that the set of complexity random points has measure 1 .

In the definition of complexity randomness we can require the set $A$ to be co-c.e. closed.

Proposition 5.11. A point $x$ is complexity random iff there exists $d \in \mathbb{N}$ such that

$$
x \in \psi^{-}(p) \Rightarrow K m(p) \geq-\log \mu\left(\psi^{-}(p)\right)-d
$$

for each computable sequence $p \in \operatorname{dom}\left(\psi^{-}\right)$.

Proof. The "only if" direction is immediate.

Suppose that $x$ is not complexity random. Then for each $d \in \mathbb{N}$, there exists a closed set $A$ such that $x \in A$ and $K m(A)<-\log \mu(A)-d$. Then $K m(A)$ is finite. Hence $A$ is co-c.e. closed. Let $p \in 2^{\omega}$ be a sequence such that $K m(A)=$ $K m(p)$ and $\psi^{-}(p)=A$. Then $x \in \psi^{-}(p)$ and $K m(p)<-\log \mu\left(\psi^{-}(p)\right)-d$.

Further we can require the set $A$ to be the complement of the finite union of base sets. Recall that $\nu^{\mathrm{fs}}$ is a notation of finite unions of base sets. For simplicity, we define $F: \subseteq 2^{*} \rightarrow \mathcal{A}$ be the set function as

$$
F(u)=F_{\nu}(u)=X \backslash \bigcup \nu^{\mathrm{fs}}(u) .
$$

Proposition 5.12. A point $x$ is complexity random iff there exists $d \in \mathbb{N}$ such that

$$
x \in F(u) \Rightarrow K m(u) \geq-\log \mu(F(u))-d
$$

for each $u \in \operatorname{dom}\left(\nu^{\mathrm{fs}}\right)$.

Proof. The "only if" direction is immediate.

Suppose that $x$ is not complexity random. Then for each $d \in \mathbb{N}$ there exists a computable sequence $p \in 2^{\omega}$ such that $x \in \psi^{-}(p)$ and $K m(p)<$ $-\log \mu\left(\psi^{-}(p)\right)-2 d$. Then there exists $u \in \operatorname{dom}\left(\nu^{\mathrm{fs}}\right)$ such that $u \prec p$ and

$$
\mu(F(u)) \leq 2^{d} \cdot \mu\left(\psi^{-}(p)\right) .
$$

Since $u \prec p$, we have $\bigcup \nu^{\mathrm{fs}}(u) \subseteq \theta(p)$ and $x \notin \bigcup \nu^{\mathrm{fs}}(u)$. Again by $u \prec p$, we have $K m(u) \leq K m(p)$. Hence $K m(u) \leq K m(p)<-\log \mu(F(u))-d$.

This proposition says that complexity randomness has always universality. 
Proposition 5.13. The set of complexity random points has measure 1.

Proof. Let $k \in \mathbb{N}$. We define a c.e. set $U=U_{k}$ as

$U=\left\{(\sigma, u):|\sigma|<-\log \mu(F(u))-k, u \in \operatorname{dom}\left(\nu^{\mathrm{fs}}\right), u \preceq v\right.$ and $\left.(\sigma, v) \in M\right\}$.

Note that the set of non-complex random sets is $\bigcap_{k} \bigcup_{\langle p, u\rangle \in U} F(u)$.

We define sets $V$ and $W$ as

$$
V=\{u:(\sigma, u) \in U \text { for some } \sigma\}, W=\{u: v \notin V \text { for all } v \prec u\} \text {. }
$$

For each $u \in W \subseteq V$, let $\sigma_{u}$ be such that $\left(\sigma_{u}, u\right) \in U$.

We claim that $\bigcup_{u \in W} F(u)=\bigcup_{(\sigma, u) \in U} F(u)$. The inclusion $\subseteq$ is immediate. We show the other inclusion. Suppose that $x \in F(u)$ for some $(\sigma, u) \in U$. Then $u \in V$. If $u \in W$ then $x \in F(u)$ for this $u \in W$. If $u \notin W$, there exists $v$ such that $v \prec u$ and $v \in W$. Then $x \in F(u) \subseteq F(v)$.

Since $W$ is prefix-free and $M$ is monotone, the set $\left\{\sigma_{u}: u \in W\right\}$ is prefixfree. Then

$$
2^{k} \mu\left(\bigcup_{(\sigma, u) \in U} F(u)\right) \leq \sum_{u \in W} 2^{k} \mu(F(u)) \leq \sum_{u \in W} 2^{-\left|p_{u}\right|} \leq 1 .
$$

Hence the set $\bigcap_{k} \bigcup_{(\sigma, u) \in U_{k}} F(u)$ has measre 0 .

\section{$5.4 \quad K$-complexity randomness}

Proposition 5.12 says that complexity randomness has a characterization by $K m(u)$ where $u$ is a string and not a sequence. Then we also consider a similar definition.

A prefix-free machine is a partical computable function whose domain is prefix-free. There exists a universal prefix-free machine $U: \subseteq 2^{*} \rightarrow 2^{*}$ and define

$$
K(\tau)=\min \{|\sigma|: U(\sigma)=\tau\} .
$$

The following is a basic tool to study complexity $K$. A $K C$ set is a c.e. set $W=\left\{\left\langle d_{i}, \tau_{i}\right\rangle: d_{i} \in \mathbb{N}, \tau_{i} \in 2^{*}\right\}_{i}$ such that $\sum_{i} 2^{-d_{i}} \leq 1$.

Theorem 5.14 (KC Theorem; Levin [13], Schnorr [21], Chaitin [4]). For a KC set $\left\{d_{i}, \tau_{i}\right\}_{i}$, there ix a prefix-free machine $M$ and strings $\sigma_{i}$ of length $d_{i}$ such that $M\left(\sigma_{i}\right)=\tau_{i}$ for all $i$ and $\operatorname{dom}(M)=\left\{\sigma_{i}: i \in \mathbb{N}\right\}$.

Definition 5.15. A point $x$ is $K$-complexity random if there exists $d \in \mathbb{N}$ such that

$$
x \in F(u) \Rightarrow K(u) \geq-\log \mu(F(u))-d
$$

for each $u \in \operatorname{dom}\left(\nu^{\mathrm{fs}}\right)$.

The notion of $K$-complexity randomness does not depend on the notation neither. 
Proposition 5.16. Let $\mathbf{X}_{1}=\left(X, \tau, \beta_{1}, \nu_{1}\right)$ and $\mathbf{X}_{2}=\left(X, \tau, \beta_{2}, \nu_{2}\right)$ be equivalent computable topological spaces. Then $K$-complexity randomness on $\mathbf{X}_{1}$ and $K$ complexity randomness on $\mathbf{X}_{2}$ coincide.

Proof. Suppose $\nu_{2} \leq \theta_{1}$. Let $F_{i}=F_{\nu_{i}}$ for $i=1,2$.

We define a partial computable function $h_{k}: \subseteq 2^{*} \rightarrow 2^{*}$ as follows. For each $k \in \mathbb{N}$ and $u \in 2^{*}$, search $v$ such that

$$
\nu_{1}^{\mathrm{fs}}(v) \subseteq \nu_{2}(u) \text { and } K(u)<-\log \mu\left(F_{1}(v)\right)-2 k .
$$

If found, let $h_{k}(u)=v$.

We define a $\mathrm{KC}$ set $L$ as

$$
L=\left\{\left\langle|\sigma|+k+1, h_{k}(U(\sigma))\right\rangle: h_{k}(U(\sigma)) \downarrow\right\}
$$

where $U$ is the universal prefix-free machine. Then

$$
\sum_{\langle n, v\rangle \in L} 2^{-n} \leq \sum_{\sigma \in \operatorname{dom}(U)} 2^{-|\sigma|-k-1} \leq 1 .
$$

Then there exists $d \in \mathbb{N}$ such that

$$
K\left(h_{k}(u)\right) \leq K(u)+k+d+1
$$

for each $u \in \operatorname{dom}\left(h_{k}\right)$.

Suppose that $x$ is not $K$-complexity random on $\mathbf{X}_{2}$. For each $k \in \mathbb{N}$, there exists $u \in \operatorname{dom}\left(\nu_{2}^{\mathrm{fs}}\right)$ such that $x \in F_{2}(u)$ and $K(u)<-\log \mu\left(F_{2}(u)\right)-2 k-$ 1. Then there exists $v \in \operatorname{dom}\left(\nu_{1}^{\mathrm{fs}}\right)$ such that $\nu_{1}^{\mathrm{fs}}(v) \subseteq \nu_{2}(u)$ and $\mu\left(F_{1}(v)\right) \leq$ $2 \mu\left(F_{2}(u)\right)$. It follows that $F_{1}(v) \supseteq F_{2}(u) \ni x$ and

$$
K(u)<-\log \mu\left(F_{2}(u)\right)-2 k-1 \leq-\log \mu\left(F_{1}(v)\right)-2 k .
$$

Hence $h_{k}(u)$ is defined. It follows that

$$
K\left(h_{k}(u)\right) \leq K(u)+k+d+1<-\log \mu\left(F_{1}\left(h_{k}(u)\right)\right)-k+d+1 .
$$

Hence $x$ is not $K$-complexity random on $\mathbf{X}_{1}$.

Since $K m(\sigma) \leq K(\sigma)+d$ for all $\sigma \in 2^{*}$ for some $d \in \mathbb{N}$, complexity randomness implies $K$-complexity randomness. Hence the following is immediate from Proposition 5.13. Here we also give an easy direct proof.

Proposition 5.17. The set of $K$-complexity random points has measure 1.

Proof. For each $k$, we define a set $A_{n}$ as

$$
A_{n}=\{u: K(u)<-\log \mu(F(u))-k\} .
$$

For each $u \in A_{k}$, we have $2^{k} \mu(F(u)) \leq 2^{-K(u)}$. Then

$$
\mu\left(\bigcup_{u \in A_{k}} F(u)\right) \leq \sum_{u \in A_{n}} \mu(F(u)) \leq 2^{-k} \sum_{u \in A_{n}} 2^{-K(u)} \leq 2^{-k}
$$

for each $k$. It follows that $\bigcap_{n} \bigcup_{u \in A_{k}} F(u)$ has measure 0 . Hence the set of non- $K$-complexity random points has measure 0 . 


\subsection{When they coincide}

Complexity randomness and $K$-complexity randomness are randomness notions that can be defined on any computable topological space with any computable measure. However Martin-Löf randomness does not coincide with each of them in general.

Example 5.18. Consider the lower unit interval $\mathbf{I}_{<}$in Example 2.3 and the measure $\mu$ such that $\mu((q, 1])=1-q$ where $q \in \mathbb{Q} \cap[0,1]$. Then $\mu$ is computable. The set of non-ML-random points is $\{1\}$. In contrast the set of non-complexity random points is $\{0\}$. The set of non-K-complexity random points is also $\{0\}$.

We show that $\mathrm{SCT}_{3}$ is a sufficient condition for the coincidence.

Theorem 5.19. Let $\mathbf{X}$ be an $\mathrm{SCT}_{3}$ space and $\mu$ be a computable measure on it. Then the following are equivalent for a point $x \in X$ :

(i) $x$ is $M L$-random.

(ii) $x$ is complexity random.

(iii) $x$ is $K$-complexity random.

The implication (ii) $\Rightarrow$ (iii) is immediate.

Proof of $(i) \Rightarrow$ (ii) of Theorem 5.19. By Lemma 4.9, there exists a double sequence $\left\{U_{n}^{u}\right\}$ of uniformly c.e. open sets and a double sequence $\left\{V_{n}^{u}\right\}$ of uniformly co-c.e. closed sets such that $U_{n}^{u} \uparrow \bigcup \nu^{\mathrm{fs}}(u)$ and $V_{n}^{u} \uparrow \bigcup \nu^{\mathrm{fs}}(u)$ for all $u \in \operatorname{dom}\left(\nu^{\mathrm{fs}}\right)$ and $U_{n}^{u} \subseteq V_{n}^{u} \subseteq \bigcup \nu^{\mathrm{fs}}(u)$ for all $n \in \mathbb{N}$ and $u \in \operatorname{dom}\left(\nu^{\mathrm{fs}}\right)$. Let $k \in \mathbb{N}$. We define a c.e. set $S_{k}=S$ of strings as

$$
S=\left\{(\sigma, u, n):|\sigma|<-\log \mu\left(X \backslash V_{n}^{u}\right)-k, u \in \operatorname{dom}\left(\nu^{\mathrm{fs}}\right), u \preceq v,(\sigma, v) \in M\right\}
$$

where $M$ is the universal monotone machine.

Let $W_{k}=\bigcup_{(\sigma, u, n) \in S}\left(X \backslash V_{n}^{u}\right)$. We will prove that $\mu\left(W_{k}\right) \leq 2^{-k}$. The argument is similar to the proof of Proposition 5.13. We define sets $A$ and $B$ as

$$
A=\{u:(\sigma, u, n) \in S \text { for some } \sigma, n\}, B=\{u: v \notin A \text { for all } v \prec u\} .
$$

For each $u \in B$, let $n_{u}$ be the smallest $n$ such that $(\sigma, u, n) \in S$ for some $\sigma$ and let $\sigma_{u}$ be such that $\left(\sigma_{u}, u, n_{u}\right) \in S$.

We claim that

$$
\bigcup_{u \in W}\left(X \backslash V_{n_{u}}^{u}\right)=\bigcup_{(\sigma, u, n) \in S}\left(X \backslash V_{n}^{u}\right) .
$$

The inclusion $\subseteq$ is immediate. We show the other direction. Suppose that $x \in X \backslash V_{n}^{u}$ for some $(\sigma, u, n) \in S$. Then $u \in A$. If $u \in B$, then $V_{n_{u}}^{u} \subseteq V_{n}^{u}$ and $x \in X \backslash V_{n_{u}}^{u}$. If $u \notin B$, then there exists $v$ such that $v \prec u$ and $v \in B$. By the construction in Lemma 4.9, we can assume

$$
V_{n}^{v} \subseteq V_{n}^{u} \text { for each } v \prec u \text {. }
$$


It follows that $V_{n_{v}}^{v} \subseteq V_{n}^{v} \subseteq V_{n}^{u}$ and $x \in X \backslash V_{n_{v}}^{v}$.

Since $B$ is prefix-free and $M$ is monotone, the set $\left\{\sigma_{u}: u \in B\right\}$ is prefix-free. Then

$$
\begin{aligned}
\mu\left(\bigcup_{(\sigma, u, n) \in S}\left(X \backslash V_{n}^{u}\right)\right) & =\mu\left(\bigcup_{u \in W}\left(X \backslash V_{n_{u}}^{u}\right)\right) \\
& \leq \sum_{u \in W} \mu\left(X \backslash V_{n_{u}}^{u}\right) \leq \sum_{u \in W} 2^{-\left|\sigma_{u}\right|-k} \leq 2^{-k} .
\end{aligned}
$$

For each $k$, let $T_{k}=\bigcup_{(p, u, n) \in S_{k}}\left(X \backslash V_{n}^{u}\right)$. Then $\left\{T_{k}\right\}$ is ML-test.

Suppose that $x$ is not complexity random. By Proposition 5.12, for each $k$, there exists $u_{0} \in \operatorname{dom}\left(\nu^{\mathrm{fs}}\right)$ such that $x \in F\left(u_{0}\right)$ and $K m\left(u_{0}\right)<-\log \mu\left(F\left(u_{0}\right)\right)-$ $k$. Let $\sigma_{0}$ be such that $K m\left(u_{0}\right)=\left|\sigma_{0}\right|, v \preceq u_{0}$ and $\left(\sigma_{0}, v\right) \in M$ for some $v$. Since $\left|\sigma_{0}\right|<-\log \mu\left(F\left(u_{0}\right)\right)-k$ and $\lim _{n} \mu\left(X \backslash V_{n}^{u_{0}}\right)=\mu\left(F\left(u_{0}\right)\right)$, there exists $n_{0}$ such that $\left|\sigma_{0}\right|<-\log \mu\left(X \backslash V_{n_{0}}^{u_{0}}\right)-k$. Then $\left(\sigma_{0}, u_{0}, n_{0}\right) \in S$. Hence

$$
x \in F\left(u_{0}\right) \subseteq X \backslash V_{n_{0}}^{u_{0}} \subseteq T_{k} .
$$

Since $k$ is arbitrary, $x$ is not ML-random.

Before giving a proof of the remaining implication, we show a lemma.

Lemma 5.20. Let $\mathbf{X}$ be an $\mathrm{SCT}_{3}$ space and $\mu$ be a computable measure on it. For each c.e. open set $W$ and $n \in \mathbb{N}$, one can compute a computable sequence $\left\{V_{m}\right\}$ of the finite unions of base sets and a computable sequence $\left\{C_{m}\right\}$ of the complements of the finite unions of base sets such that

(i) $W \subseteq \bigcup_{m} C_{m}$,

(ii) $V_{m} \subseteq C_{m}, \mu\left(C_{m}\right)-\mu\left(V_{m}\right) \leq 2^{-n-m-1}$ and

(iii) $\sum_{m} \mu\left(C_{m}\right) \leq 2 \mu(W)+2^{-n}$ for each $n$.

Proof. By Lemma 4.9, there exists a sequence $\left\{U_{k}\right\}$ oe uniformly c.e. open sets and a sequence $\left\{A_{k}\right\}$ of uniformly co-c.e. closed sets such that $U_{k} \uparrow W, A_{k} \uparrow W$ and $U_{k} \subseteq A_{k} \subseteq W$. Further we assume that $U_{k}$ is the finite union of base sets.

We claim that, for each $m$, one can compute $k=k(m)$ such that $\mu\left(A_{k}\right)-$ $\mu\left(U_{k}\right)<2^{-n-m-3}$. Note that the real $\mu\left(U_{k}\right)$ is approximated from below and $\mu\left(A_{k}\right)$ is approximated from above. Furthere $\mu(W)-\mu\left(U_{k}\right) \rightarrow 0$ as $k \rightarrow \infty$, we have $\mu\left(A_{k}\right)-\mu\left(U_{k}\right) \rightarrow 0$. Hence we can compute such $k$. We assume that $k(m)<k(m+1)$ for all $m$.

We define $B_{k}=B_{k(m)}$ for each $m$ as follows. Since $A_{k}$ is co-c.e. closed set and $\mu\left(A_{k}\right)-\mu\left(U_{k}\right)<2^{-n-m-3}$, there exists $B_{k}$ such that $A_{k} \subseteq B_{k}, B_{k}$ is the complement of the finite union of base sets and $\mu\left(B_{k}\right)-\mu\left(U_{k}\right)<2^{-n-m-3}$. It should be noted that $B_{k}$ is defined only when $k=k(m)$ for some $m$.

We define a computable sequence $\left\{V_{m}\right\}$ of the finite unions of base sets and a computable sequence $\left\{C_{m}\right\}$ of the complements of the finite unions of base sets as follows. Let $V_{0}=U_{k(0)}, C_{0}=B_{k(0)}$,

$$
V_{m}=U_{k(m)} \backslash B_{k(m-1)} \text { and } C_{m}=B_{k(m)} \backslash U_{k(m-1)}
$$


for all $m \geq 1$. It should be noted that $X \backslash C_{m}=\left(X \backslash B_{k(m)}\right) \cup U_{k(m-1)}$ is the finite union of base sets for each $m$.

We show $W \subseteq \bigcup_{m} C_{m}$. Since $U_{k} \uparrow W$, it suffices to show that

$$
U_{k(m)} \subseteq C_{0} \cup \cdots \cup C_{m}
$$

by induction over $m$. The case of $m=0$ is true by the definition. Suppose that the inclusion (3) is true for $m-1$. Then

$$
U_{k(m)} \subseteq A_{k(m)} \subseteq B_{k(m)} \subseteq C_{m} \cup U_{k(m-1)} \subseteq C_{0} \cup \cdots \cup C_{m}
$$

Hence the inclusion (3) is true for $m$.

We prove $V_{m} \subseteq C_{m}$ for all $m$. Suppose that $x \in V_{m}$ Then $x \in U_{k(m)}$ and $x \notin B_{k(m-1)}$. Since $U_{k(m)} \subseteq B_{k(m)}, x \in B_{k(m)}$ and $X \notin U_{k(m-1)}$. Hence $x \in C_{m}$

Next we show $\mu\left(C_{m}\right)-\mu\left(V_{m}\right) \leq 2^{-n-m-1}$. Since $U_{k(m-1)} \subseteq U_{k(m)} \subseteq B_{k(m)}$, we have

$$
\mu\left(C_{m}\right)=\mu\left(B_{k(m)} \backslash U_{k(m-1)}\right)=\mu\left(B_{k(m)}\right)-\mu\left(U_{k(m-1)}\right) .
$$

We also have

$$
\begin{aligned}
\mu\left(V_{m}\right) & =\mu\left(U_{k(m)} \backslash B_{k(m-1)}\right)=\mu\left(U_{k(m)}\right)-\mu\left(U_{k(m)} \cap B_{k(m-1)}\right) \\
& \geq \mu\left(U_{k(m)}\right)-\mu\left(B_{k(m-1)}\right) .
\end{aligned}
$$

It follows that

$$
\begin{aligned}
\mu\left(C_{m}\right)-\mu\left(V_{m}\right) & \leq \mu\left(B_{k(m)}\right)-\mu\left(U_{k(m-1)}\right)-\mu\left(U_{k(m)}\right)+\mu\left(B_{k(m-1)}\right) \\
& \leq 2^{-n-m-3}+2^{-n-(m-1)-3}<2^{-n-m-1} .
\end{aligned}
$$

Finally we prove $\sum_{m} \mu\left(B_{m}\right) \leq 2 \mu(W)+2^{-n}$. For $m \geq 1$, $C_{m}=B_{k(m)} \backslash U_{k(m-1)}=\left(B_{k(m)} \backslash A_{k(m)}\right) \uplus\left(A_{k(m)} \backslash A_{k(m-1)}\right) \uplus\left(A_{k(m-1)} \backslash U_{k(m-1)}\right.$ and

$$
\begin{aligned}
\mu\left(C_{m}\right) & <2^{-n-m-3}+\mu\left(A_{k(m)} \backslash A_{k(m-1)}\right)+2^{-n-(m-1)-3} \\
& <\mu\left(A_{k(m)} \backslash A_{k(m-1)}\right)+2^{-n-m-1}
\end{aligned}
$$

Then

$$
\sum_{m} \mu\left(C_{m}\right) \leq \mu\left(B_{k(0)}\right)+\sum_{m} \mu\left(A_{k(m)} \backslash A_{k(m-1)}\right)+\sum_{m} 2^{-n-m-1} .
$$

The first term is less than or equal to $\mu(W)$. The second term is also less than or equal to $\mu(W)$. The third term is equal to $2^{-n}$. 
Proof of $($ iii $) \Rightarrow$ (i) of Theorem 5.19. Let $\left\{W_{n}\right\}$ be a ML-test. By Lemma 5.20, there exists a computable suquence $\left\{V_{\langle n, m\rangle}\right\}$ of the finite unions of base sets and a computable sequence $\left\{C_{\langle n, m\rangle}\right\}$ of the complements of the finite unions of base sets such that

$$
\begin{aligned}
& W_{2 n+6} \subseteq \bigcup_{m} C_{\langle n, m\rangle}, V_{\langle n, m\rangle} \subseteq C_{\langle n, m\rangle}, \\
& \mu\left(C_{\langle n, m\rangle}\right)-\mu\left(V_{\langle n, m\rangle}\right) \leq 2^{-2 n-5-m-1}=2^{-2 n-m-6} \\
& \sum_{m} \mu\left(C_{\langle n, m\rangle}\right) \leq 2 \mu\left(W_{2 n+6}\right)+2^{-2 n-5} \leq 2^{-2 n-5}+2^{-2 n-5}=2^{-2 n-4}
\end{aligned}
$$

for each $n$.

We construct a KC set as follows. Let

$$
L_{1}=\{\langle n+m+3,\langle n, m\rangle\rangle: n, m \in \mathbb{N}\} .
$$

Note that $\sum_{n, m} 2^{-n-m-3}=1 / 2$. We construct another $\mathrm{KC}$ set $L_{2}$. Since $V_{\langle n, m\rangle} \subseteq C_{\langle n, m\rangle}$, we have $-\log \mu\left(C_{\langle n, m\rangle}\right) \leq-\log \mu\left(V_{\langle n, m\rangle}\right)$. Since $C_{\langle n, m\rangle}$ is c.e. open and $V_{\langle n, m\rangle}$ is co-c.e. closed, $-\log \mu\left(V_{\langle n, m\rangle}\right)$ is approximated from above and $-\log \mu\left(C_{\langle n, m\rangle}\right)$ is approximated from below. Then the relation $-\log \mu\left(V_{\langle n, m\rangle}\right)+$ $\log \mu\left(C_{\langle n, m\rangle}\right)<1$ is semi-decidable. If the relation holds, there exists an integer $b$ such that $b-1<-\log \mu\left(C_{\langle n, m\rangle}\right) \leq-\log \mu\left(V_{\langle n, m\rangle}\right)<b+1$, which is equivalent to

$$
2^{-b-1}<\mu\left(V_{\langle n, m\rangle}\right) \leq \mu\left(C_{\langle n, m\rangle}\right)<2^{-b+1} .
$$

Note that such $b$ can be found effectively. Let $b(n, m)$ be the integer $b$ if found. Let

$$
L_{2}=\{\langle b(n, m)-n-1,\langle n, m\rangle\rangle: b(n, m) \text { is defined }\}
$$

Then

$$
\sum_{b(n, m) \downarrow} 2^{-b(n, m)+n+1} \leq \sum_{n, m} 2^{n+2} \mu\left(C_{\langle n, m\rangle}\right) \leq \sum_{n} 2^{n+2} \cdot 2^{-2 n-4}=1 / 2 .
$$

Hence $L_{1} \cup L_{2}$ is a $\mathrm{KC}$ set.

Let $f: \subseteq 2^{*} \rightarrow \mathbb{N}$ be the prefix-free machine constructed from $L_{1} \cup L_{2}$ by $\mathrm{KC}$ theorem. Recall that $C_{\langle n, m\rangle}$ is the comlement of the finite union of base sets. Then there exists a computable sequence $\left\{u_{\langle n, m\rangle}\right\}$ such that $F\left(u_{\langle n, m\rangle}\right)=C_{\langle n, m\rangle}$. Let $h(\sigma)=u_{f(\sigma)}$. Then $h$ is a prefix-free machine.

Suppose that $x \in X$ is not ML-random. Since $x \in \bigcap_{n} W_{n}$ and $W_{2 n+6} \subseteq$ $\bigcup_{m} C_{\langle n, m\rangle}$, there exists $m$ such that $x \in C_{\langle n, m\rangle}=F\left(u_{\langle n, m\rangle}\right)$ for each $n$.

Suppose that $b(n, m)$ is defined. Then there exists $\sigma \in 2^{*}$ such that $|\sigma|=$ $b(n, m)-n-1<-\log \mu\left(C_{\langle n, m\rangle}\right)-n$, which implies

$$
K_{h}\left(u_{\langle n, m\rangle}\right) \leq-\log \mu\left(F\left(u_{\langle n, m\rangle}\right)\right)-n .
$$

Suppose that $b(n, m)$ is not defined and $\mu\left(C_{\langle n, m\rangle}\right) \leq 2^{-2 n-m-4}$. Then $-\log \mu\left(C_{\langle n, m\rangle}\right)-n-1 \geq n+m+3$. Note that there exists $\sigma \in 2^{*}$ such that $|\sigma|=n+m+3$ and $h(\sigma)=u_{\langle n, m\rangle}$. Then

$$
K_{h}\left(u_{\langle n, m\rangle}\right) \leq n+m+3 \leq-\log \mu\left(F\left(u_{\langle n, m\rangle}\right)\right)-n-1
$$


Suppose that $b(n, m)$ is not defined and $\mu\left(C_{\langle n, m\rangle}\right)>2^{-2 n-m-4}$. Then

$$
\mu\left(V_{\langle n, m\rangle}\right)>\mu\left(C_{\langle n, m\rangle}\right)-2^{-2 n-m-6} \geq \mu\left(C_{\langle n, m\rangle}\right) / 2 .
$$

It follows that

$$
-\log \mu\left(C_{\langle n, m\rangle}\right) \leq \mu\left(V_{\langle n, m\rangle}\right)<-\log \mu\left(C_{\langle n, m\rangle}\right)+1 .
$$

This is a contradiction.

Hence $x$ is not $K$-complexity random.

\subsection{When they do not coincide}

The condition of $\mathrm{SCT}_{3}$ is a sufficient condition for the coincidnece between MLrandomness and complexity randomness. We prove that we can not weaken the condition to $\mathrm{SCT}_{2}$. To prove it, we use the following computable topological space.

Consider the unit interval $\mathbf{I}=([0,1], \tau, \beta, \nu)$ in Example 2.3. Let $a$ be a real in $[0,1]$. We write $I_{a}=[0,1] \backslash\{a\}$. Let

$$
\nu_{a}(\langle u, v\rangle)= \begin{cases}\nu(v) & \text { if } u=\lambda \text { and } v \in \operatorname{dom}(\nu), \\ \nu(v) \cap I_{a} & \text { if } u \neq \lambda \text { and } v \in \operatorname{dom}(\nu),\end{cases}
$$

where $\lambda$ is the empty string. Let $\beta_{a}=\left\{\nu_{a}(u): u \in \operatorname{dom}\left(\nu_{a}\right)\right\}$. Since $I_{a}$ is open on $\mathbf{I}$, the topology generated by the base $\beta_{a}$ coincides with $\tau$.

Proposition 5.21. The 4-tuple $\mathbf{I}_{a}=\left([0,1], \tau, \beta_{a}, \nu_{a}\right)$ is $\mathrm{SCT}_{2}$. Further the following are equivalent.

(i) $\mathbf{I}_{a}$ is $\mathrm{SCT}_{3}$.

(ii) a is $\delta_{a}$-computable.

(iii) $a$ is $\delta$-computable.

Note that $\mathbf{I}_{a}$ is $T_{3}$ for all $a \in[0,1]$.

Proof. We prove that $\mathbf{I}_{a}$ is a computable topological space. Clearly $\mathbf{I}_{a}$ is an effective topological space clearly. Note that $\operatorname{dom}\left(\nu_{a}\right)$ is computable. Let $S \subseteq$ $\left(\Sigma^{*}\right)^{3}$ be a c.e. set such that

$$
\nu(u) \cap \nu(v)=\bigcup\{\nu(w):(u, v, w) \in S\}
$$

for all $u, v \in \operatorname{dom}(\nu)$. Let $S_{a} \subseteq\left(\Sigma^{*}\right)^{3}$ be the c.e. set such that

$$
\left(\left\langle u_{1}, u_{2}\right\rangle,\left\langle v_{1}, v_{2}\right\rangle,\left\langle w_{1}, w_{2}\right\rangle\right) \in S_{a} \Longleftrightarrow\left(u_{2}, v_{2}, w_{2}\right) \in S \wedge w_{1}=\lambda
$$

if $u_{1}=v_{1}=\lambda$ and

$$
\left(\left\langle u_{1}, u_{2}\right\rangle,\left\langle v_{1}, v_{2}\right\rangle,\left\langle w_{1}, w_{2}\right\rangle\right) \in S_{a} \Longleftrightarrow\left(u_{2}, v_{2}, w_{2}\right) \in S \wedge w_{1} \neq \lambda
$$


if $u_{1} \neq \lambda$ or $v_{1} \neq \lambda$. Then $S_{a}$ satisfies (1) in Definition 2.1.

Since $\mathbf{I}$ is $\mathrm{SCT}_{2}$ and $\beta \subseteq \beta_{a}, \mathbf{I}_{a}$ is also $\mathrm{SCT}_{2}$.

(ii) $\Rightarrow\left(\right.$ iii) Suppose that the real $a$ is $\delta_{a}$-computable on $\mathbf{I}_{a}$. Then the set $\{\langle u, v\rangle \in$ $\left.\Sigma^{*}: a \in \nu_{a}(\langle u, v\rangle)\right\}$ is c.e. Since $a \notin I_{a}$, we have

$$
a \in \nu_{a}(\langle u, v\rangle) \Longleftrightarrow u=\lambda \text { and } a \in \nu(v)
$$

Hence $\left\{v \in \Sigma^{*}: a \in \nu(v)\right\}$ is c.e. It follows that $a$ is $\delta$-computable.

(iii) $\Rightarrow$ (ii) This is proved by tracking back the proof of (ii) $\Rightarrow$ (iii).

(iii) $\Rightarrow$ (i) Consider a base set $W$ that has the form $W=(p, q) \cap I_{a}$ where $p, q \in \mathbb{Q}$. Since $a$ is $\delta$-computable, it is decidable whether $a \in(p, q)$ or not. If not, $W=((p, a) \cap[0,1]) \cup((a, q) \cap[0,1]$. Now it is easy to construct $R$ and $r$ in Definition 2.5.

(i) $\Rightarrow$ (ii) Note that $I_{a}$ is a base set. By Lemma 4.9, one can construct a computable sequence $\left\{U_{n}\right\}$ of finite unions of base sets and a computable sequence $\left\{V_{n}\right\}$ of closed sets such that $U_{n} \uparrow I_{a}, V_{n} \uparrow I_{a}$ and $U_{n} \subseteq V_{n} \subseteq I_{a}$ for all $n$. Since $I_{a}$ is not closed and $V_{n}$ is closed, $U_{n} \subsetneq I_{a}$.

Consider the diameter of a set $A \subseteq[0,1]$ as

$$
D(A)=\sup \{|x-y|: x, y \in A\} .
$$

Since $U_{n}$ is a finite union of base sets, $D\left([0,1] \backslash U_{n}\right)$ is computable. Since $U_{n} \subsetneq$ $I_{a},[0,1] \backslash U_{n}$ has at least two elements and $D\left([0,1] \backslash U_{n}\right)>0$. Since $U_{n} \uparrow I_{a}$, $D\left([0,1] \backslash U_{n}\right) \rightarrow 0$ as $n \rightarrow 0$. It follows that $a$ is a $\delta$-computable real.

Proposition 5.22. There exists an $\mathrm{SCT}_{2}$ and $T_{3}$ space with a computable measure on which ML-randomness and complexity randomness does not coincide.

Proof. Let $a$ be a ML-random real on $\mathbf{I}$. We prove that $a$ is ML-random but not complexity random on $\mathbf{I}_{a}$ with the Lebesgue measure $\mu$.

The co-c.e. closed set $\{a\}$ has measure 0 and $a \in\{a\}$. Hence $a$ is not complexity random.

Suppose $a$ is not ML-random on $\mathbf{I}_{a}$. Then there exists a ML-test $\left\{U_{n}\right\}$ with $a \in \bigcap_{n} U_{n}$. Since $\left\{U_{n}\right\}$ is uniformly c.e. open, there exist computable sequences $\left\{u_{i}^{n}\right\}$ and $\left\{v_{i}^{n}\right\}$ such that

$$
U_{n}=\bigcup\left\{\nu_{a}\left(\left\langle u_{i}^{n}, v_{i}^{n}\right\rangle\right): i \in \mathbb{N}\right\} .
$$

Let

$$
V_{n}=\bigcup\left\{\nu_{a}\left(\left\langle u_{i}^{n}, v_{i}^{n}\right\rangle\right): u_{i}^{n}=\lambda \text { and } i \in \mathbb{N}\right\} \text {. }
$$

Then $V_{n} \subseteq U_{n}$. Since $a \in \bigcap_{n} U_{n}$, there exists $i$ such that $a \in \nu_{a}\left(\left\langle u_{i}^{n}, v_{i}^{n}\right\rangle\right)$ for each $n$. If $u_{i}^{n} \neq \lambda, a \in \nu\left(v_{i}^{n}\right) \cap I_{a}$, which is a contradiction. Hence the $i$ should satisfy $u_{i}^{n}=\lambda$ for each $n$. It follows that $a \in \bigcap_{n} V_{n}$.

However $\left\{V_{n}\right\}$ is a ML-test on $\mathbf{I}$. Hence this contradicts to the fact that $a$ is ML-random on $\mathbf{I}$. 


\section{Discussion}

First we studied computability of measures on a computable topological space. We generalize the result by Gács to that the space of measres on a computable topological space is another computable topological space. Then computability of points on the space concindes with computability defined in Schröder [23]. Hence this is the right definition of computability of measures.

Next we studied Martin-Löf randomness on a computable topological space with a computable measure. We showed that there is not a universal test in general, and ML-randomness and complexity randomness do not coincide in general. A sufficient condition is $\mathrm{SCT}_{3}$. However we can not weaken the condition of $\mathrm{SCT}_{3}$ to $\mathrm{CT}_{2}$ for the existence of a universal test and to $\mathrm{SCT}_{2}$ for the coincidence between ML-randomness and complexity randomness. Hence a computable metric space is a rather general space on which ML-randomness is a natural notion and may be the best to which we can generalize ML-randomness as a natural randomness notion.

\section{Acknowledgement}

The author thanks anonymous referees for for useful comments. This work was partly supported by GCOE, Kyoto University and JSPS KAKENHI 23740072.

\section{References}

[1] V. Bogachev. Measure theory. Springer, 2007.

[2] V. Bosserhoff. Notions of probabilistic computability on represented spaces. Journal of Universal Computer Science, 14(6):956-995, 2008.

[3] V. Brattka, P. Hertling, and K. Weihrauch. A tutorial on computable analysis. New Computational Paradigms, pages 425-491, 2008.

[4] G. J. Chaitin. A theory of program size formally identical to information theory. Journal of the Association for Computing Machinery, 22:329-340, 1975.

[5] S. B. Cooper. Computability theory. CRC Press, 2004.

[6] R. Downey and D. R. Hirschfeldt. Algorithmic Randomness and Complexity. Springer, Berlin, 2010.

[7] A. Edalat. A computable approach to measure and integration theory. Information and Computation, 207(5):642-659, 2009.

[8] P. Gács. Uniform test of algorithmic randomness over a general space. Theoretical Computer Science, 341:91-137, 2005. 
[9] T. Grubba, M. Schröder, and K. Weihrauch. Computable metrization. Mathematical Logic Quarterly, 53(4-5):381-395, 2007.

[10] P. Hertling and K. Weihrauch. Random elements in effective topological spaces with measure. Information and Computation, 181(1):32-56, 2003.

[11] M. Hoyrup and C. Rojas. Computability of probability measures and Martin-Löf randomness over metric spaces. Information and Computation, 207(7):830-847, 2009.

[12] M. Hoyrup, C. Rojas, and K. Weihrauch. Computability of the RadonNikodym derivative. Models of Computation in Context, pages 132-141, 2011.

[13] L. A. Levin. Some Theorems on the Algorithmic Approach to Probability Theory and Information Theory. PhD thesis, Moscow, 1971.

[14] L. A. Levin. On the notion of a random sequence. Soviet Mathematics Doklady, 14:1413-1416, 1973.

[15] L. A. Levin. Laws of information conservation (nongrowth) and aspects of the foundation of probability theory. Problems of Information Transmission, 10:206-210, 1974.

[16] M. Li and P. Vitányi. An introduction to Kolmogorov complexity and its applications. Graduate Texts in Computer Science. Springer-Verlag, New York, third edition edition, 2009.

[17] P. Martin-Löf. The Definition of Random Sequences. Information and Control, 9(6):602-619, 1966.

[18] A. Nies. Computability and Randomness. Oxford University Press, USA, 2009.

[19] P. Odifreddi. Classical Recursion Theory, volume 1. North-Holland, 1990.

[20] P. Odifreddi. Classical Recursion Theory, volume 2. North-Holland, 1999.

[21] C. P. Schnorr. Process complexity and effective random tests. Journal of Computer and System Sciences, 7:376-388, 1973.

[22] M. Schröder. Effective metrization of regular spaces. In K.-I. Ko, A. Nerode, M. B. Pour-el, K. Weihrauch, and J. Wiedermann, editors, Computability and Complexity in Analysis, volume 235 of Informatik Berichte, pages 6380, FernUniversität, August 1998. CCA Workshop, Brno, Czech Republic, August, 1998.

[23] M. Schröder. Admissible Representations for Probability Measures. Mathematical Logic Quarterly, 53(4-5):431-445, 2007. 
[24] R. I. Soare. Recursively enumerable sets and degrees. Perspectives in Mathematical Logic. Springer, Berlin, 1987.

[25] J. Ville. Étude critique de la notion de collectif. Gauthier-Villars, 1939.

[26] R. von Mises. Grundlagen der Wahrscheinlichkeistrechnung. Mathematische Zeitschrift, 5:52-99, 1919.

[27] V. G. Vovk. The law of the iterated logarithm for random Kolmogorov, or chaotic, sequences. Theory of Probability and Its Applications, 32:413-425, 1987.

[28] K. Weihrauch. Computably Regular Topological Spaces. Submitted.

[29] K. Weihrauch. Computability on the Probability Measureson the Borel Sets of the Unit Interval. Theoretical Computer Science, 219(1-2):421-437, 1999.

[30] K. Weihrauch. Computable Analysis: an introduction. Springer, Berlin, 2000 .

[31] K. Weihrauch. Computable Separation in Topology, from $T_{0}$ to $T_{3}$. In $C C A, 2009$.

[32] K. Weihrauch. Computable Separation in Topology, from $T_{0}$ to $T_{2}$. Journal of Universal Computer Science, 16(18):2733-2753, 2010.

[33] K. Weihrauch and T. Grubba. Elementary Computable Topology. Journal of Universal Computer Science, 15(6):1381-1422, 2009.

[34] S. Willard. General Topology. Addison-Wesley, 1970.

[35] Y. Wu and K. Weihrauch. A computable version of the Daniell-Stone theorem on integration and linear functionals. Theoretical Computer Science, 359(1-3):28-42, 2006.

[36] A. K. Zvonkin and L. A. Levin. The complexity of finite objects and the development of the concepts of information and randomness by means of the theory of algorithms. Russian Mathematical Surveys, 25(6):83-124, 1970 . 